\title{
Simplex stochastic collocation with ENO-type stencil selection for robust uncertainty quantification
}

\author{
Jeroen A.S. Witteveen ${ }^{\mathrm{a}, \mathrm{b}, *}$, Gianluca Iaccarino ${ }^{\mathrm{a}}$ \\ ${ }^{a}$ Center for Turbulence Research, Stanford University, Building 500, Stanford, CA 94305-3035, USA \\ ${ }^{\mathrm{b}}$ Center for Mathematics and Computer Science (CWI), Science Park 123, 1098XG Amsterdam, The Netherlands
}

\section{A R T I C L E I N F O}

\section{Article history:}

Received 1 September 2011

Received in revised form 27 December 2012

Accepted 31 December 2012

Available online 11 January 2013

\section{Keywords:}

Essentially non-oscillatory

Stencil selection

Stochastic collocation

Uncertainty quantification

Discontinuities

\begin{abstract}
A B S T R A C T
Multi-element uncertainty quantification approaches can robustly resolve the high sensitivities caused by discontinuities in parametric space by reducing the polynomial degree locally to a piecewise linear approximation. It is important to extend the higher degree interpolation in the smooth regions up to a thin layer of linear elements that contain the discontinuity to maintain a highly accurate solution. This is achieved here by introducing Essentially Non-Oscillatory (ENO) type stencil selection into the Simplex Stochastic Collocation (SSC) method. For each simplex in the discretization of the parametric space, the stencil with the highest polynomial degree is selected from the set of candidate stencils to construct the local response surface approximation. The application of the resulting SSC-ENO method to a discontinuous test function shows a sharper resolution of the jumps and a higher order approximation of the percentiles near the singularity. SSC-ENO is also applied to a chemical model problem and a shock tube problem to study the impact of uncertainty both on the formation of discontinuities in time and on the location of discontinuities in space.
\end{abstract}

(c) 2013 Elsevier Inc. All rights reserved.

\section{Introduction}

Resolving discontinuities in stochastic problems is important, since they can lead to high sensitivities with respect to input uncertainties. They can also result in oscillatory approximations and, consequently, in the prediction of non-zero probabilities for unphysical realizations such as negative static pressures. In order to avoid these problems, the polynomial interpolation degree can locally be reduced to a linear approximation to avoid overshoots at the discontinuity in a multi-element uncertainty quantification (UQ) approach. In this context, two points are essential to maintain a highly accurate solution despite the locally first degree approximation. Firstly, the region in which the interpolation is reduced to a piecewise linear function should be as small as possible. This means that the samples need to be concentrated around the discontinuity to pinpoint its location. Secondly, the higher degree interpolation in the smooth regions should be extended as close as possible up to the discontinuity to maintain high order accuracy near the singularity. These two objectives are achieved here by introducing an Essentially Non-Oscillatory (ENO) type stencil selection into the Simplex Stochastic Collocation (SSC) method.

The ENO scheme has been developed by Harten and Osher [13] as a robust spatial discretization in the finite volume method (FVM) for deterministic Computational Fluid Dynamics (CFD) [14]. In that field, the robust approximation of discontinuities is critical for resolving shock waves and contact surfaces in the flow field. Therefore, it was proposed by Abgrall [1] and Barth [6] to use shock-capturing FVM to discretize also the parametric space to obtain robust approximations for

\footnotetext{
* Corresponding author at: Center for Turbulence Research, Stanford University, Building 500, Stanford, CA 94305-3035, USA

E-mail addresses: jeroen.witteveen@cwi.nl (J.A.S. Witteveen), jops@stanford.edu (G. Iaccarino).
} 
stochastic CFD. These FVM discretizations of the combined physical and parametric space use the ENO and Weighted Essentially Non-Oscillatory (WENO) [16] schemes. Since FVM has originally been developed for the three-dimensional physical space, the direct extension of FVM to these high dimensional parametric spaces can, however, be inefficient due to the curse-of-dimensionality. Because of the different nature of the parametric space, there are also no physical fluxes between the cells in the stochastic directions, which form the basis of FVM. Therefore, we follow a different approach to extend the robustness of FVM to parametric space. We reformulate the robustness principles of FVM in terms of the parametric space and develop new UQ methods that satisfy these concepts in the stochastic dimensions. This approach has the advantages that it maintains the FVM robustness in combination with the effectiveness of specifically designed methods for UQ, which can also be used with other spatial discretizations than FVM. In this way, we have previously introduced, for instance, the Total Variation Diminishing (TVD) [12,31], Extremum Diminishing (ED) [15,33], and Local Extremum Diminishing (LED) [15,35] principles into UQ and proposed the Essentially Extremum Diminishing (EED) [34] concept.

The ENO spatial discretization [13] achieves an essentially non-oscillatory approximation of the solution of hyperbolic conservation laws. Non-oscillatory means, in this context, that the number of local extrema in the solution does not increase with time. The ENO scheme obtains this property using an adaptive-stencil approach with a uniform polynomial degree for reconstructing the spatial fluxes. Each spatial cell $X_{j}$ is assigned $r$ stencils $\left\{S_{j i}\right\}_{i=1}^{r}$ of degree $p$, all of which include the cell $X_{j}$ itself. Out of this set of candidate stencils $\left\{S_{j, i}\right\}$, the stencil $S_{j}$ is selected for cell $X_{j}$ that results in the interpolation $w_{j}(\mathbf{x})$ which is smoothest in some sense based on an indicator of smoothness $\mathrm{IS}_{j, i}$. In this way, a cell next to a discontinuity is adaptively given a stencil consisting of the smooth part of the solution, which avoids Gibbs-like oscillations in physical space. Attention has been paid to the efficient implementation of ENO schemes by Shu and Osher [24,25]. Fig. 1 shows an example of the ENO stencil selection in a FVM discretization of a discontinuity in one spatial dimension using piecewise quadratic polynomials.

ENO-type stencil selection is here used in the SSC multi-element UQ method to obtain an accurate approximation of discontinuities in parametric space. Multi-element UQ methods discretize the stochastic dimensions using multiple subdomains comparable to spatial discretizations in physical space. These local methods $[3,19,28]$ can be based on Stochastic Galerkin (SG) projections of Polynomial Chaos (PC) expansions [10,36] in each of the subdomains. Other methods $[2,8,17]$ use a Stochastic Collocation (SC) approach $[4,37]$ to construct the local polynomial approximations based on sampling at quadrature points in the elements. These methods commonly use sparse grids of Gauss quadrature rules in hypercube subdomains combined with solution-based refinement measures for resolving nonlinearities. Because of the hypercube elements, these methods are most effective in capturing discontinuities that are aligned with one of the stochastic coordinates.

In contrast, the SSC method $[35,34]$ is based on a simplex tessellation of the parametric space with sampling points at the vertexes of the simplex elements. The polynomial approximation in the simplexes $\Xi_{j}$ is built using higher degree interpolation stencils $S_{j}$, with local polynomial degree $p_{j}$, consisting of samples in the vertexes of surrounding simplexes. The degree $p_{j}$ is controlled by a Local Extremum Conserving (LEC) limiter, which reduces $p_{j}$ and the stencil size to avoid overshoots in the interpolation of the samples where necessary. The limiter, therefore, leads to a non-uniform polynomial degree that reduces to a linear interpolation in simplexes which contain a discontinuity and that increases away from singularities. SSC employs adaptive refinement measures based on the hierarchical surplus and the geometrical properties of the simplexes to identify the location of discontinuities. However, the limiter can result in an excessive reduction of the polynomial degree also at significant distances away from a discontinuity. Since the polynomial degree affects the refinement criteria, this can also deteriorate the effectiveness of the refinement to sharply resolve singularities.

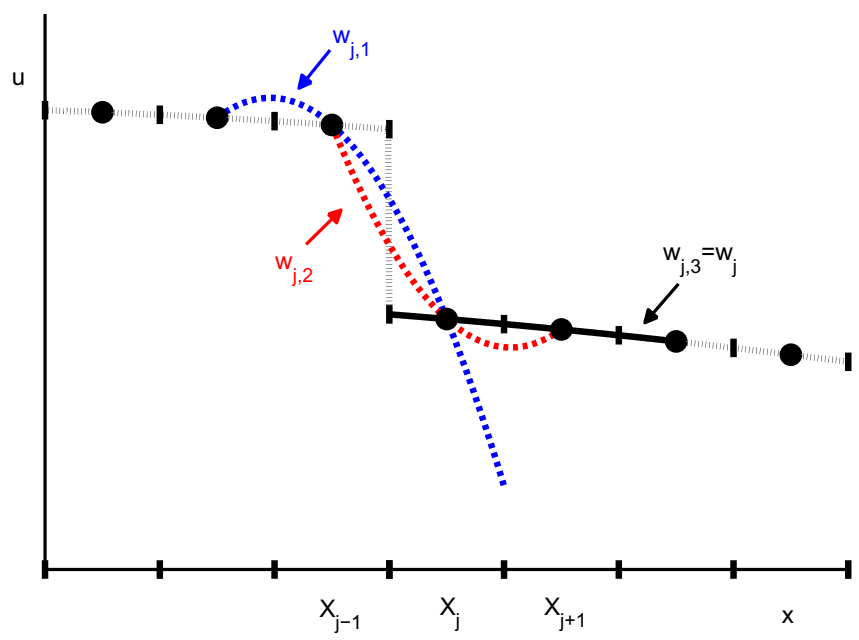

Fig. 1. ENO stencil selection for the quadratic reconstruction $w_{j}(x)$ in the spatial cell $X_{j}$ out of the candidates $\left\{w_{j, 1}, w_{j, 2}, w_{j, 3}\right\}$ for cell-centered FVM in one physical dimension. 
In order to obtain a more accurate solution of nonlinear response surfaces, ENO-type stencil selection is introduced into the SSC method. For each simplex $\Xi_{j}, r_{j}$ stencils $\left\{S_{j, i}\right\}_{i=1}^{r_{j}}$ are constructed that contain $\Xi_{j}$, and the stencil $S_{j}$ is selected that results in the smoothest interpolation $w_{j}(\xi)$. The polynomial degree $p_{j, i}$ of the candidate stencils $S_{j, i}$ that are accepted by the LEC limiter, is used as the indicator of smoothness $I S_{j, i}$. A simplex $\Xi_{j}$ near a discontinuity, therefore, achieves a higher order approximation by assigning $\Xi_{j}$ a higher degree interpolation stencil $S_{j}$ that does not contain the discontinuity. The higher polynomial degree $p_{j}$ leads also to lower values of the refinement measures in $\Xi_{j}$, which restricts the refinement more to the simplexes that contain the discontinuity. The stencil selection does not affect the linear approximation in the latter simplexes, since the LEC limiter rejects the higher degree stencils that contain these elements.

The developed SSC-ENO method is applied to a chemical model problem and a shock tube problem. The chemical model problem is used to analyze the impact of uncertainty in initial conditions on the formation of a discontinuity in time. The initial-value problem with a normal input distribution was proposed by Reagan et al. [23] as a model involving nonlinearity and bifurcation behavior, which are both characteristic for chemical systems. They used it to determine the effectiveness of global PC formulations for problems resulting in a large coefficient of variation $(\mathrm{CoV})$. The model was also considered by Le Maître et al. [20] to compare the global PC expansion with their multi-resolution scheme. They both conclude that the problem is too challenging for global representations. A system with similar behavior describing the movement of a particle subject to a potential field and a friction force with an uncertain initial position was also studied by Le Maître et al. [18]. A different class of unsteady models that involves the long time integration problem is treated, for instance, in $[9,21,30]$.

The shock tube problem is considered to study the solution of a system of hyperbolic conservation laws with uncertain discontinuity locations in space. Uncertainty analysis of hyperbolic systems has received relatively little attention [11]. The example involves Sod's Riemann problem [26] for the Euler equations of inviscid compressible gas dynamics. This Riemann problem was used by Poëtte et al. [22] to illustrate their PC method based on an entropic variable in an example with steep fronts and shocks. The problem was also studied by Tryoen et al. [27] using upwinding in a multi-resolution approach based on local SG projections of the PC expansion. Abgrall [1] used shock tube like test cases to demonstrate the application of his method to the Euler equations.

The efficient implementation of ENO-type stencil selection in the SSC method is discussed in Section 2. In Section 3, the effectiveness of the resulting SSC-ENO approach is analyzed for a discontinuous test function. The chemical model problem and the shock tube problem are considered in Sections 4 and 5, respectively. The conclusions are summarized in Section 6.

\section{Simplex stochastic collocation with stencil selection}

An efficient algorithm for the SSC-ENO interpolation stencil selection is developed below. The implementation and the effect on the adaptive refinement are also discussed.

\subsection{Simplex stochastic collocation}

The SSC method [35,34] is a non-intrusive multi-element UQ approach that solves the following computational problem subject to $n_{\xi}$ second-order random parameters $\xi=\left\{\xi_{1}, \ldots, \xi_{n_{\xi}}\right\}$ in parametric space $\Xi \subset \mathbb{R}^{n_{\xi}}$

$$
\mathcal{L}(\mathbf{x}, t, \xi ; u(\mathbf{x}, t, \xi))=\mathcal{S}(\mathbf{x}, t, \xi)
$$

with output quantity of interest $u(\mathbf{x}, t, \xi)$, space $\mathbf{x}$, and time $t$. The latter two arguments are dropped from here onto simplify the notation. The response surface $u(\xi)$ is approximated by a piecewise polynomial function $w(\xi)$ using a PC $[10,36]$ expansion $w_{j}(\xi)$ in each of the simplexes $\Xi_{j}$

$$
w_{j}(\xi)=\sum_{i=0}^{P_{j}} c_{j, i} \boldsymbol{\Psi}_{j, i}(\xi)
$$

for $\xi \in \Xi_{j}$, where $\boldsymbol{\Psi}_{j, i}$ are the basis polynomials, $c_{j, i}$ are the coefficients, and $P_{j}+1=\left(n_{\xi}+p_{j}\right) ! /\left(n_{\xi} ! p_{j} !\right)$ is the number of expansion terms, with $p_{j}$ the local polynomial degree. The coefficients $c_{j, i}$ are computed by interpolating a stencil $S_{j}$ out of the $n_{s}$ samples $\mathbf{v}=\left\{v_{1}, \ldots, v_{n_{s}}\right\}$, with $v_{k}=u\left(\xi_{k}\right)$ and $k=1, \ldots, n_{s}$, at the sampling points $\xi_{k}$ in the vertexes of the $n_{\mathrm{e}}$ simplexes $\Xi_{j}$ with $j=1, \ldots, n_{\mathrm{e}}$. The deterministic simulations for $v_{k}$ are assumed to be computationally intensive such that they dominate the computational cost of the uncertainty analysis.

For a piecewise linear interpolation $w_{j}(\xi)$ with $p_{j}=1$, the stencil $S_{j}=\left\{\xi_{k_{j, 0}}, \ldots, \xi_{k_{j, N_{i}}}\right\}$ consists of the $N_{j}+1=n_{\xi}+1$ vertexes of $\Xi_{j}$, with $k_{j, l} \in\left\{1, \ldots, n_{s}\right\}$ for $j=1, \ldots, n_{\mathrm{e}}$ and $l=0, \ldots, N_{j}$. Higher degree stencils $S_{j}$ with $N_{j} \geqslant P_{j}$ are constructed by adding vertexes $\xi_{k}$ of surrounding simplexes to $S_{j}$ according to a nearest neighbor search based on the Euclidean distance to the center of the simplex $\Xi_{j}$ in parametric space $\Xi$. The center of $\Xi_{j}$ is defined as the average of the vertex locations of $\Xi_{j}$. We use $N_{j}=P_{j}$ here, but the interpolation can be constructed using a least squares approximation for $N_{j}>P_{j}$. The notation is visualized in Fig. 2 for an example of a response surface approximation in a two-dimensional parametric space with $n_{\xi}=2$.

The polynomial degree $p_{j}$ is chosen as high as possible with respect to the total number of available samples $n_{\mathrm{s}}$ with $N_{j}+1 \leqslant n_{\mathrm{s}}$. The Local Extremum Conserving (LEC) limiter reduces the stencil size $N_{j}+1$, and $p_{j}$, when $w_{j}(\xi)$ does not satisfy 


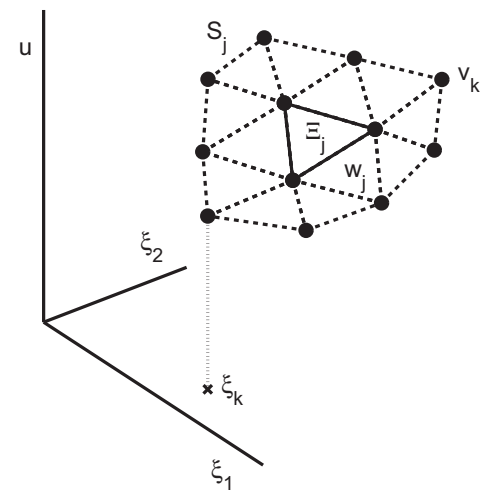

Fig. 2. Approximation of the response surface $u(\xi)$ by the interpolation $w_{j}(\xi)$ of the samples $v_{k}$ at a stencil $S_{j}$ of sampling points $\xi_{k}$ for the simplex $\Xi_{j}$ in a two-dimensional parametric space with $\xi=\left\{\xi_{1}, \xi_{2}\right\}$.

$$
\min _{\xi \in \Xi_{j}} w_{j}(\xi)=\min \mathbf{v}_{j} \wedge \max _{\xi \in \Xi_{j}} w_{j}(\xi)=\max \mathbf{v}_{j}
$$

with $\mathbf{v}_{j}=\left\{v_{k_{j, 0}}, \ldots, v_{k_{j, n}}\right\}$ the samples in $\Xi_{j}$. The limiter (3) is applied to all simplexes in $S_{j}$ and always holds for $p_{j}=1$. For additional robustness, the polynomial degree can be reduced by one more order than required by (3), if $p_{j}>1$. The $i$ th moment $\mu_{\mathrm{u}_{i}}$ of $u(\xi)$ is then computed by evaluating the following integrals using Monte Carlo integration with $n_{\mathrm{mc}} \gg n_{\mathrm{s}}$ integration points

$$
\mu_{\mathrm{u}_{i}} \approx \sum_{j=1}^{n_{\mathrm{e}}} \int_{\Xi_{j}} w_{j}(\xi)^{i} f_{\xi}(\xi) \mathrm{d} \xi,
$$

weighted by the input probability density $f_{\xi}(\xi)$ of $\xi$. For more detailed information is referred to [35,34].

\subsection{Interpolation stencil selection}

The nearest neighbor construction of the interpolation stencils $S_{j}$ combined with the LEC limiter (3) results in one stencil $S_{j}$ for each simplex $\Xi_{j}$. If the stencils $S_{j}$ are not restricted to the nearest neighbor sampling points $\xi_{k}$, then multiple stencils $S_{j, i}$ may be possible for simplex $\Xi_{j}$ that satisfy the limiter. The stencil $S_{j, i}$ that leads to the smoothest interpolation $w_{j, i}(\xi)$ is then selected for a more accurate approximation of $u(\xi)$.

The first $n_{\xi}+1$ sampling points $\xi_{k}$ of each stencil $S_{j, i}=\left\{\xi_{k_{j, 0}}, \ldots, \xi_{k_{j, n}}\right\}$ consist of the vertexes of $\Xi_{j}$. This stencil corresponds to the piecewise linear interpolation. The higher degree stencils of $N_{j, i}+1$ sampling points

$$
S_{j, i}=\left\{\boldsymbol{\xi}_{k_{j, 0}}, \ldots, \boldsymbol{\xi}_{k_{j, n_{\xi}}}, \ldots, \boldsymbol{\xi}_{k_{j, N, i}}\right\}
$$

can be constructed by adding, in principle, any combination of $N_{j, i}-n_{\xi}$ samples for any $p_{j, i}$ out of the remaining $\xi_{k}$, with $k \in\left\{1, \ldots, n_{s}\right\} \backslash\left\{k_{j, 0}, \ldots, k_{j, n_{\xi}}\right\}$ and each sampling point appearing only once in the stencil $S_{j, i}$. Out of these stencils, only a set of $r_{j}$ candidate stencils $\left\{S_{j, i}\right\}_{i=1}^{r_{j}}$ is accepted of which the interpolation $w_{j, i}(\xi)$ satisfies the limiter. The stencil $S_{j} \in\left\{S_{j, i}\right\}$ for $\Xi_{j}$ is selected based on the non-uniform polynomial degree $p_{j, i}$ as the indicator of smoothness IS $_{j, i}=p_{j, i}$

$$
S_{j}=S_{j, i}, \quad \text { with } i=\underset{i^{*} \in\left\{1, \ldots, r_{j}\right\}}{\arg \max } p_{j, i^{*}} .
$$

If multiple stencils have the same smoothness, then out of these stencils the one with the minimum average Euclidean distance of the sampling points $\xi_{k}$ to the center of $\Xi_{j}$ is chosen.

A two-dimensional example is given in Fig. 3 of the stencil selection for $\Xi_{j}$ close to a discontinuity, of which the location is denoted by the diagonal line. The nearest neighbor stencil $S_{j}$ for $\Xi_{j}$ only leads to a quadratic interpolation $w_{j}(\xi)$ with $N_{j}+1=6$, since higher degree stencils cross the discontinuity and are rejected by the limiter. On the other hand, stencil selection can result in $S_{j}$, with a higher polynomial degree, that contains all sampling points $\xi_{k}$ in the smooth region at one side of the discontinuity.

\subsection{Efficient implementation}

Constructing all possible stencils $S_{j, i}$ for all simplexes $\Xi_{j}$ can become impractical as its complexity increases binomially with the number of samples $n_{s}$. Therefore, we restrict the stencil selection to a subset of these stencils by employing the multi-element character of the approach. We allow only nearest neighbor stencils of other simplexes that contain $\Xi_{j}$ to be assigned to $\Xi_{j}$. 


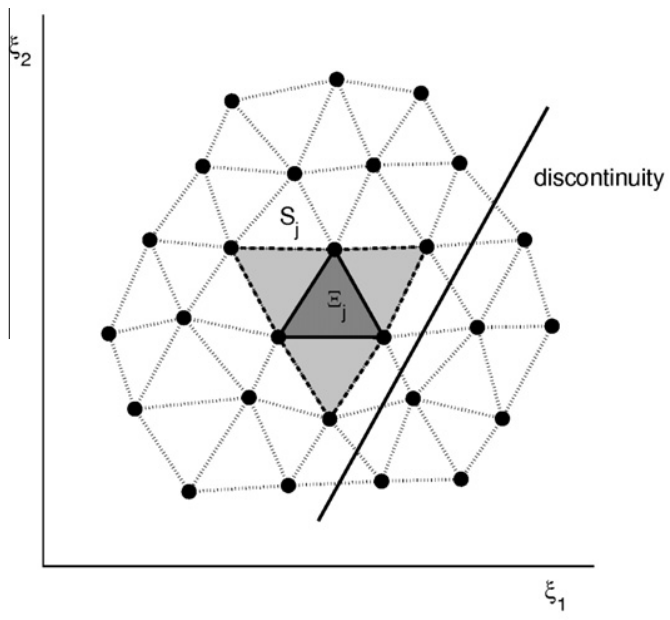

(a) Nearest neighbor stencil

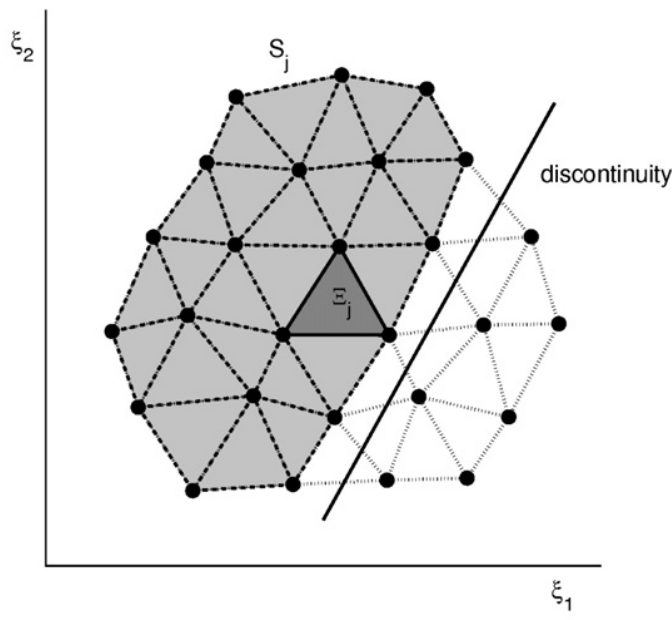

(b) Selected stencil

Fig. 3. Selection of the interpolation stencil $S_{j}$ for the simplex $\Xi_{j}$ near a discontinuity in a two-dimensional parametric space.

To that end, the nearest neighbor stencils $\tilde{S}_{j}$, with interpolation $\tilde{w}_{j}(\xi)$ and degree $\tilde{p}_{j}$, are first constructed for each $\Xi_{j}$ as described in Section 2.1. This results in a set of $n_{\mathrm{e}}$ stencils $\left\{\tilde{S}_{j}\right\}_{j=1}^{n_{\mathrm{e}}}$. Next, it needs to be determined for each $\tilde{S}_{j}$ which simplexes $\Xi_{i}$ are part of the stencil. A stencil $\tilde{S}_{j}$ is considered to contain $\Xi_{i}$, if $\tilde{S}_{j}$ contains all vertexes $\left\{\boldsymbol{\xi}_{k_{i 0}}, \ldots, \boldsymbol{\xi}_{k_{i n}}\right\}$ of $\Xi_{i}$. A set of $\tilde{r}_{j}$ candidate stencils $\left\{\tilde{S}_{j, i}\right\}_{i=1}^{\tilde{r}_{j}}$ for $\Xi_{j}$ is then collected from the nearest neighbor stencils that contain $\Xi_{j}$. The stencil $S_{j}=\tilde{S}_{j, i}$, and the interpolation $w_{j}(\xi)=\tilde{w}_{j, i}(\xi)$, with the highest degree $\tilde{p}_{j, i}$ is selected from $\left\{\tilde{S}_{j, i}\right\}_{i=1}^{\tilde{r}_{j}}$ as in (6). If none of the stencils $\left\{\tilde{S}_{j, i}\right\}$ has a higher degree than the nearest neighbor stencil $\tilde{S}_{j}$, i.e. $\tilde{p}_{j, i} \leqslant \tilde{p}_{j}$ for all $i=1, \ldots, \tilde{r}_{j}$, then the original stencil $\tilde{S}_{j}$ is automatically maintained.

This efficient SSC-ENO stencil selection algorithm results in virtually no additional computational costs compared to SSC with nearest neighbor stencils, since no additional stencils or interpolations are constructed. The algorithm can only improve the polynomial degree with $p_{j} \geqslant \tilde{p}_{j}$. Fig. 4 shows the adoption of the nearest neighbor stencil of another simplex $\Xi_{i}$ by $\Xi_{j}$ in the two-dimensional example. Because the resulting stencil $S_{j}$ is asymmetrical with respect to $\Xi_{j}$, it leads to a higher polynomial degree $p_{j}$ than its nearest neighbor stencil of Fig. 3(a). The efficiently selected stencil does not necessarily contain all the sampling points on one side of the discontinuity.

\subsection{Solution-adaptive refinement measures}

The initial discretization of the parametric space consists of a simplex tessellation of sampling points at the corners of a hypercube parametric space and one sample in the interior, see Fig. 5(a) for a two-dimensional example on the domain

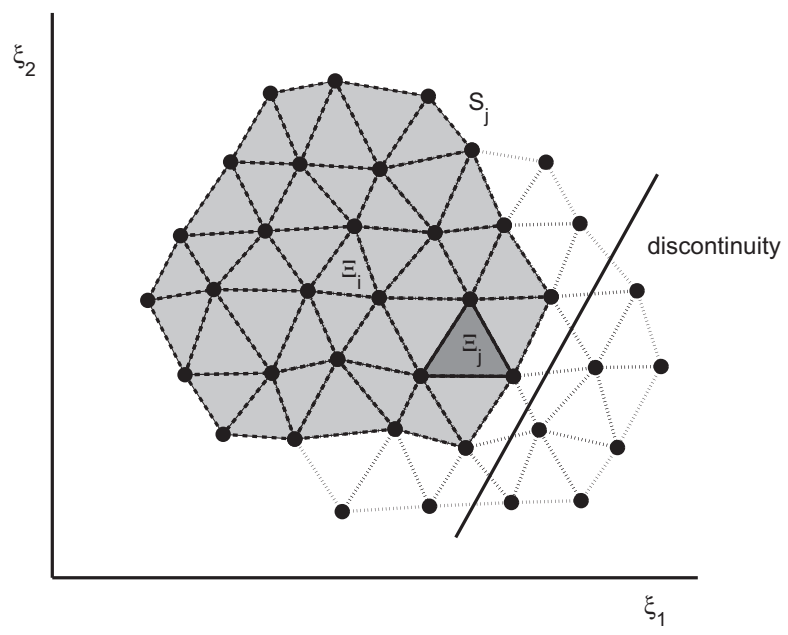

Fig. 4. Efficiently selected stencil $S_{j}$ for the simplex $\Xi_{j}$ by adopting the nearest neighbor stencil of the simplex $\Xi_{i}$ in a two-dimensional parametric space. 


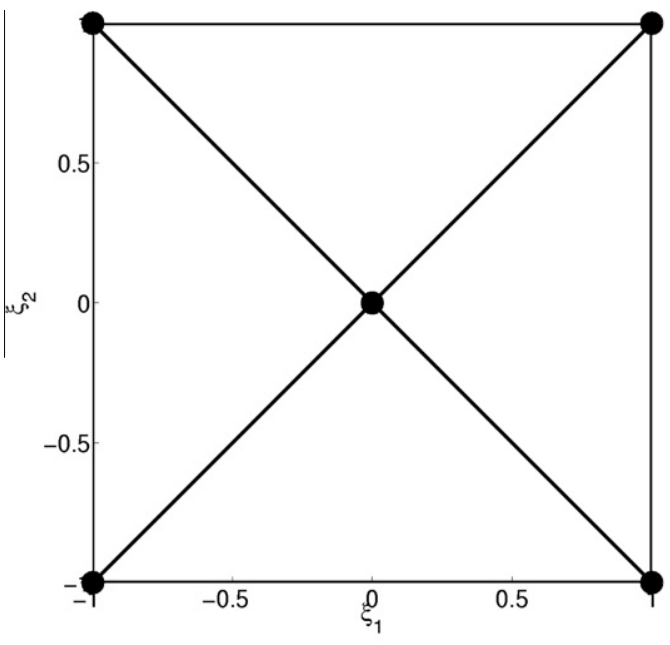

(a) Initial discretization

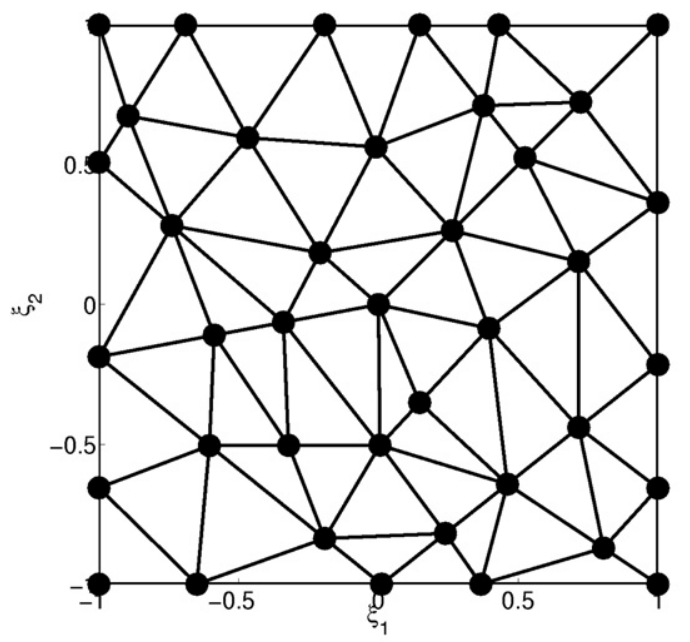

(b) Discretization with $n_{\mathrm{s}}=40$, measure $\bar{\Xi}$

Fig. 5. Simplex tessellation of the two-dimensional parametric space $\Xi$, where the sampling points $\xi_{k}$ and the simplexes $\Xi_{j}$ are denoted by the closed circles and the lines, respectively.

$[-1,1]^{n_{\xi}}$. The approximation converges by refining $\Xi_{j}$ with the highest value of a refinement measure in each refinement step. The simplex is here refined by adding a sampling point $\xi_{k_{\text {new }}}$ on the longest edge of $\Xi_{j}$ to be able to capture also discontinuities that cross the edges of the parametric space.

Consider $\xi_{k_{j, 0}}$ and $\xi_{k_{j, 1}}$ to be the endpoints of the longest edge of $\Xi_{j}$. Then $\xi_{k_{\text {new }}}$ is given a random location on the edge

$$
\xi_{k_{\text {new }}}=(\theta+(1-2 \theta) U) \xi_{k_{j, 0}}+(1-(\theta+(1-2 \theta) U)) \xi_{k_{j, 1}},
$$

with $U$ a uniform random number on $[0,1]$ and $\theta=\frac{1}{3}$ for a sufficient spread of the samples. The randomness is used for efficient sampling in multiple dimensions and for $n_{\xi}=1$ the new sampling point is deterministically placed at the center of the element with $\theta=\frac{1}{2}$. The discretization is updated by computing the sample $v_{k_{\text {new }}}=u\left(\xi_{k_{\text {new }}}\right)$ and by making a Delaunay triangulation of the sampling points. The sample $v_{k_{\text {new }}}$ can also be incorporated by splitting all simplexes that contain the edge $\left(\xi_{k_{i 0}}, \boldsymbol{\xi}_{k_{11}}\right)$ into two new simplexes with $\boldsymbol{\xi}_{k_{\text {new }}}$ one of their vertexes.

The following two solution-based refinement measures, $\varepsilon_{j}$ and $e_{j}$, are derived in $[35,34]$ based on the hierarchical surplus $\tilde{\varepsilon}_{j}$ and the geometrical properties of the simplexes

$$
\varepsilon_{j}=\bar{\Omega}_{j} \hat{\varepsilon}_{j}^{2}, \quad e_{j}=\bar{\Omega}_{j} \bar{\Xi}_{j}^{2 O_{j}},
$$

with

$$
\hat{\varepsilon}_{j}=\frac{\tilde{\varepsilon}_{j}}{\max \mathbf{v}-\min \mathbf{v}}\left(\frac{\bar{\Xi}_{j}}{\bar{\Xi}_{k^{*}, \text { ref }}}\right)^{O_{j}} .
$$

The probability $\bar{\Omega}_{j}$ and the normalized volume $\bar{\Xi}_{j}$ of $\Xi_{j}$ are defined as

$$
\bar{\Omega}_{j}=\int_{\Xi_{j}} f_{\xi}(\xi) \mathrm{d} \xi, \quad \bar{\Xi}_{j}=\frac{1}{\bar{\Xi}} \int_{\Xi_{j}} \mathrm{~d} \xi
$$

with $\bar{\Xi}=\sum_{j=1}^{n_{e}} \bar{\Xi}_{j}$ and $\bar{\Xi}_{k^{*}, \text { ref }}$ a reference volume, see $[35,34]$. The local theoretical order of convergence $O_{j}$ is given by

$$
O_{j}=\frac{p_{j}+1}{n_{\xi}} .
$$

Both measures $\varepsilon_{j}$ and $e_{j}$ depend on the local polynomial degree $p_{j}$ through expression (11). The stencil selection decreases the measures $\varepsilon_{j}$ and $e_{j}$ in the simplexes in which the solution is smooth, since $\bar{\Xi}_{j} / \bar{\Xi}_{k^{*} \text {,ref }}<1$ and $\bar{\Xi}_{j}<1$. The measure $e_{j}$ leads to solution-based refinement through the reduction of $p_{j}$ at discontinuities by the limiter. It is a more reliable measure in multiple stochastic dimensions than $\varepsilon_{j}$, since $\varepsilon_{j}$ is based on the hierarchical surplus $\tilde{\varepsilon}_{j}$ in a single discrete point. The size $\bar{\Xi}_{j}$ of the simplexes can be used as the refinement measure in order to obtain uniform or volumetric refinement, see Fig. 5(b) for an example with $n_{\mathrm{s}}=40$. 


\subsection{SSC-ENO algorithm summary}

The efficient implementation of the SSC-ENO interpolation algorithm consists of the following four steps that are performed for $j=1, \ldots, n_{\mathrm{e}}$. It replaces the interpolation step in the refinement procedure of the nearest neighbor SSC method as described in [34]:

1. Construct the nearest neighbor stencil $\tilde{S}_{j}$ for interpolation $\tilde{w}_{j}(\xi)$ with polynomial degree $\tilde{p}_{j}$ in all simplexes $\Xi_{j}$;

2. Collect the set of $\tilde{r}_{j}$ candidate stencils $\left\{\tilde{S}_{j, i}\right\}_{i=1}^{\tilde{r}_{j}}$ from the nearest neighbor stencils, with corresponding $\tilde{w}_{j, i}(\xi)$ and $\tilde{p}_{j, i}$, that contain $\Xi_{j}$;

3. Select $S_{j}=\tilde{S}_{j, i}$ that results in the highest polynomial degree $p_{j}=\tilde{p}_{j, i}$ for $\Xi_{j}$;

4. Update $w_{j}(\xi)=\tilde{w}_{j, i}(\xi)$ if $S_{j} \neq \tilde{S}_{j}$ in $\Xi_{j}$;

The stencil selection algorithm is essentially valid for arbitrary dimensionality.

\section{Multidimensional discontinuous test function}

In order to isolate the effect of the introduced stencil selection, results of the SSC-ENO method are compared to those of SSC without stencil selection. A test function with discontinuities that are not aligned with the grid is considered up to five dimensions. The impact of the refinement measures on the comparison is reported in Appendix A.

\subsection{Test function}

This example requires both a robust interpolation of the multiple sharp discontinuities of different strength intersecting each other and a higher order approximation of the smooth parts of the solution. The response $u(\xi)$ contains two Heaviside step functions and a smooth background function

$$
u(\xi)=u_{\text {hat }}(\xi)+u_{\text {back }}(\xi)
$$

with

$$
u_{\text {hat }}(\xi)=H\left(\frac{\xi \cdot \xi^{*}}{\sqrt{\xi^{*} \cdot \xi^{*}}}+\xi_{1}^{*}\right)-c_{\text {hat }} H\left(\frac{\xi \cdot \xi \prime}{\sqrt{\xi \prime \cdot \xi \prime}}+\xi_{1} \prime\right),
$$

which resembles a hat function in one dimension. The secondary jump has a strength of $c_{\text {hat }}=0.25$. Here $H$ is the following Heaviside function defined as

$$
u_{\text {step }}(\xi)=H\left(\frac{\xi \cdot \xi^{*}}{\sqrt{\xi^{*} \cdot \xi^{*}}}+\xi_{1}^{*}\right)= \begin{cases}0, & \frac{\xi \cdot \xi^{*}}{\sqrt{\xi^{*} \cdot \xi^{*}}}+\xi_{1}^{*}<0 \\ 1, & \text { otherwise. }\end{cases}
$$

The background function $u_{\text {back }}(\xi)$ is

$$
u_{\text {back }}(\xi)=\frac{1}{2}+\frac{1}{\pi} \arctan \left(c \xi_{1}\right)
$$

which is normalized to a range between 0 and 1 , with parameter value $c=0.1$. The vectors of arbitrary numbers $\xi^{*}=\left\{\xi_{1}^{*}, \ldots, \xi_{n_{\xi}}^{*}\right\} \in[-0.5 ; 0.5]^{n_{\xi}}$ and $\xi \prime=\left\{\xi_{1}, \ldots, \xi_{n_{\xi}}\right\} \in[-0.5 ; 0.5]$ give the two discontinuities arbitrary locations and orientations. The random parameters $\xi$ are uniformly distributed on the domain $U(-1,1)$.

The quality of the response surface approximation is assessed by considering the root mean square (RMS) error $\varepsilon_{\text {rms }}$ between $w(\xi)$ and $u(\xi)$. The error $\varepsilon_{\text {rms }}$ is evaluated using the weighted Monte Carlo integration used for computing the statistical moments

$$
\varepsilon_{\mathrm{rms}}=\sqrt{\frac{1}{n_{\mathrm{mc}}} \sum_{k=1}^{n_{\mathrm{mc}}}\left(w\left(\xi_{\mathrm{mc}_{k}}\right)-u\left(\xi_{\mathrm{mc}_{k}}\right)\right)^{2}}
$$

with $n_{\mathrm{mc}}$ the number of Monte Carlo integration points $\xi_{\mathrm{mc}_{\mathrm{k}}}$. The different strength of the steps is taken into account by measure $\varepsilon_{j}$ through the hierarchical surpluses, which leads approximately to an order of magnitude smaller error than for $e_{j}$ in Fig. 6 for $n_{\xi}=1$ and two orders of magnitude compared to the uniform refinement measure $\bar{\Xi}_{j}$. Measure $e_{j}$ resolves both jumps equally well, since it is based on the local polynomial degree and the LEC limiter reduces the interpolation to first order at both discontinuities. Nonetheless, we mostly use measure $e_{j}$ in the following, since it is a more reliable refinement criterion in multiple stochastic dimensions.

Fig. 7(a) shows the response surface in two dimensions and SSC-ENO with measure $e_{j}$ and $n_{\mathrm{s}}=150$ resolves both discontinuities equally well in Fig. 7(b). Starting from the initial discretization, the adaptive refinement measure $e_{j}$ automatically 


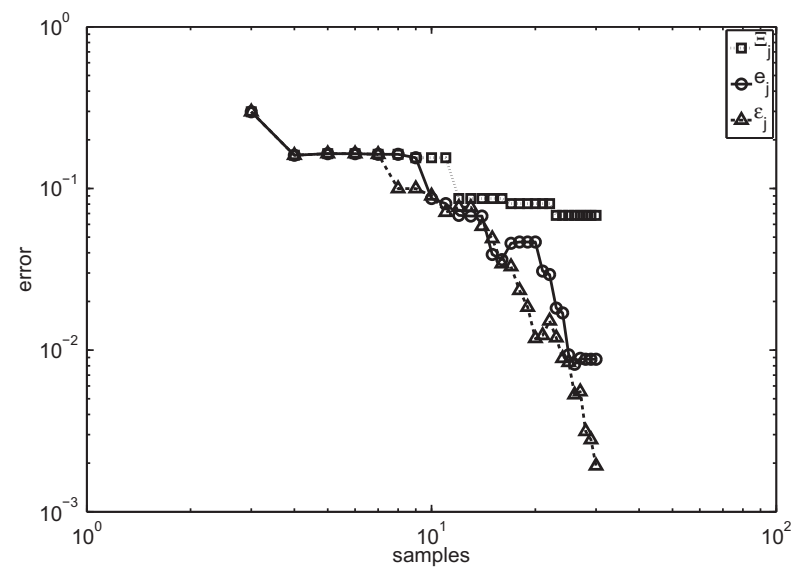

Fig. 6. Error convergence of SSC-ENO for the test function.

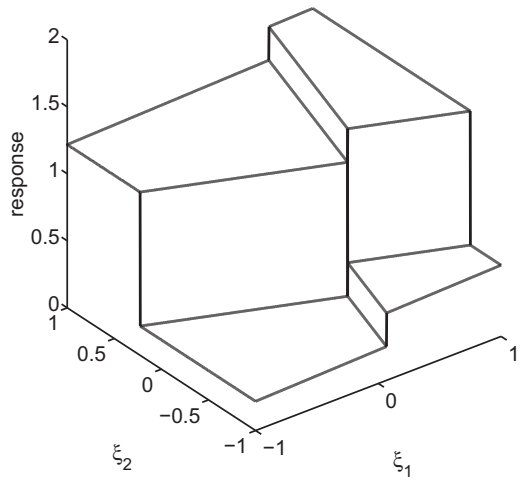

(a) Response surface

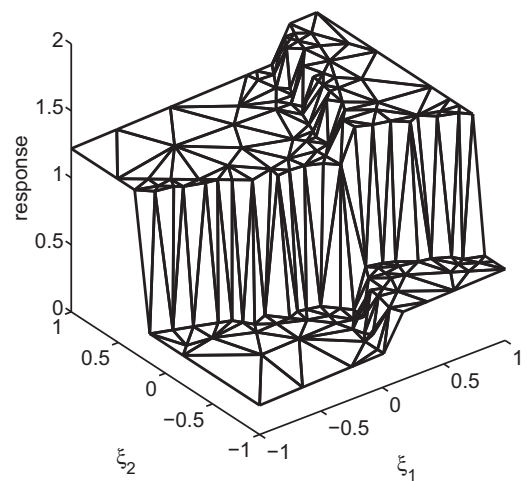

(b) SSC-ENO, measure $e_{j}, n_{\mathrm{s}}=150$

Fig. 7. Response surface approximation for the test function.

refines the simplex elements to a smaller size near the discontinuities to find their location. Farther away from the steps, the simplexes maintain a larger size.

Fig. 8 shows that SSC-ENO results in a higher concentration of samples and smaller simplexes near the discontinuities in discretization of the parametric space $\Xi$ than SSC. The sampling points, denoted by the closed circles, are clustered significantly closer to the discontinuity locations, given by the dashed diagonal lines, for SSC-ENO than for SSC. This leads to a sharper resolution of the discontinuities with smaller simplexes for SSC-ENO at equal computational costs in terms of the sample size. The more effective refinement by SSC-ENO is caused by the higher polynomial degree $p_{j}$ in the simplexes that do not contain the discontinuities. SSC-ENO results in a linear interpolation in only the small simplexes that contain the discontinuities to avoid overshoots.

The adaptive refinement measure $e_{j}$ does, however, not distinguish between the two different jump strengths, because the LEC limiter reduces the polynomial degree at both discontinuities to $p_{j}=1$. Therefore, measure $e_{j}$ places an equal number of sampling points at the strong discontinuity and the weaker one with strength $c_{\text {hat }}=0.25$. The other adaptive measure $\varepsilon_{j}$ can distinguish the strength of the discontinuities based on the hierarchical surpluses, but $\varepsilon_{j}$ is less reliable in multiple stochastic dimensions. The limitations of both measures, $e_{j}$ and $\varepsilon_{j}$, can be avoided by combining them into one mixed refinement criterion $e_{j}^{*}$

$$
e_{j}^{*}=\alpha e_{j}+(1-\alpha) \varepsilon_{j}=\bar{\Omega}_{j}\left(\alpha \bar{\Xi}_{j}^{2 \mathcal{O}_{j}}+(1-\alpha) \hat{\varepsilon}_{j}^{2}\right)
$$

with mixing parameter $\alpha \in[0,1]$, and $\bar{\Xi}_{j}$ and $\hat{\varepsilon}_{j}$ both scaled by the parametric space size $\bar{\Xi}$ and the sample range $(\max \mathbf{v}-\min \mathbf{v})$, respectively. SSC-ENO with mixed refinement measure $e_{j}^{*}$ and $\alpha=0.99$ leads in Fig. 9 to a different level of refinement for the two discontinuities with different strength. The weaker step is less refined, such that the stronger discontinuity can be resolved by an appreciable denser number of samples than in Fig. 8(b) for the same computational budget 


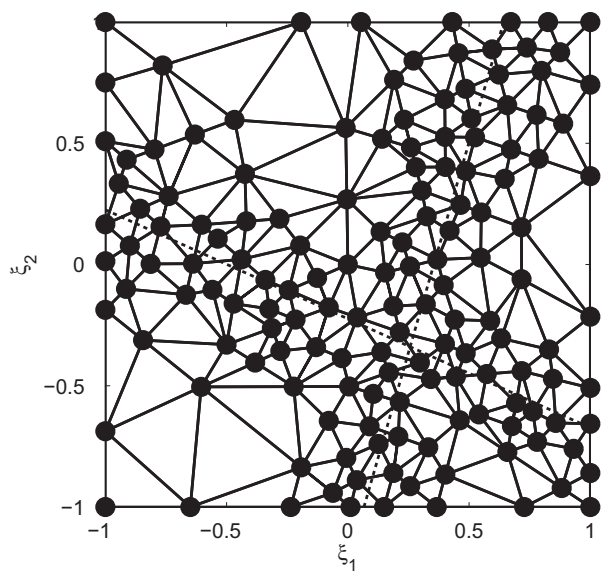

(a) SSC

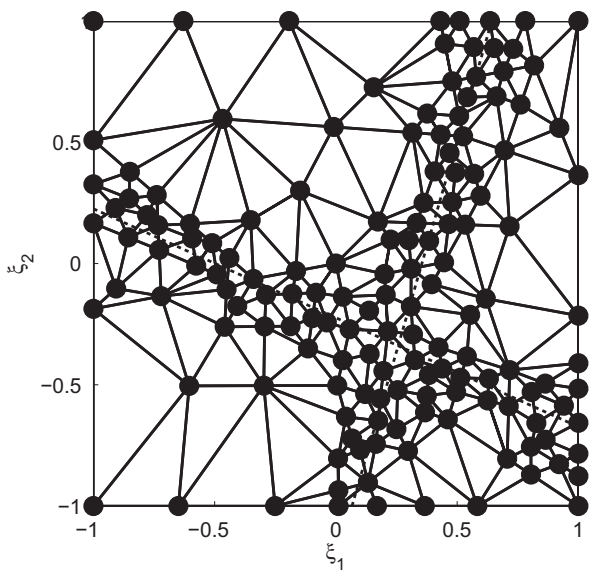

(b) SSC-ENO

Fig. 8. Discretization of the parametric space $\Xi$ with the refinement measure $e_{j}$ and $n_{\mathrm{s}}=150$ for the test function, where the simplexes and the sampling points are denoted by the lines and the closed circles, respectively, and the dashed lines show the locations of the discontinuities.

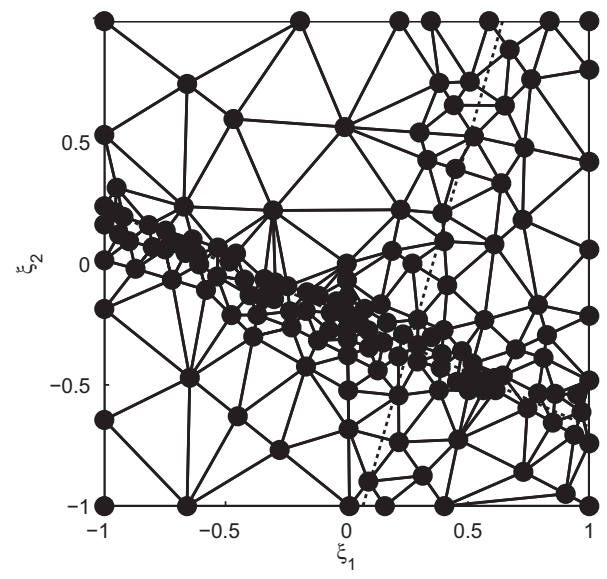

(a) Discretization

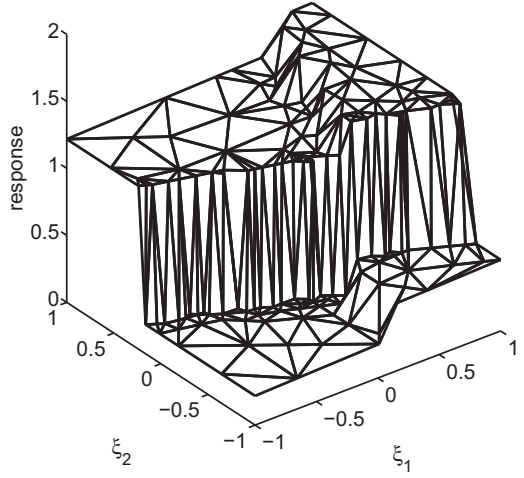

(b) Response surface approximation

Fig. 9. SSC-ENO with the mixed refinement measure $e_{j}^{*}, \alpha=0.99$, and $n_{\mathrm{s}}=150$ for the test function.

of $n_{\mathrm{s}}=150$. In the response surface approximation of Fig. 9(b), it can be seen that the larger jump is resolved more accurately at the expense of a coarser approximation of the smaller jump than in Fig. 7(b). The SSC-ENO method has also been applied successfully to test functions with non-planar discontinuities.

\subsection{Higher dimensions}

The effect of the accurate resolution by SSC-ENO of the discontinuities in (12) on the approximation error is considered here up to five dimensions for $c_{\text {hat }}=0$. The mean $\mu_{\mathrm{u}}$ and the standard deviation $\sigma_{\mathrm{u}}$ of SSC and SSC-ENO compared to a Monte

Table 1

Approximation of the mean by SSC and SSC-ENO with the refinement measure $e_{j}$ and $n_{\xi}=\{1,2,3\}$ compared to the reference solution for the test function.

\begin{tabular}{|c|c|c|c|c|c|c|}
\hline \multirow[t]{2}{*}{$n_{\xi}$} & \multirow[t]{2}{*}{$n_{\mathrm{s}}$} & \multicolumn{3}{|l|}{ Mean $\mu_{\mathrm{u}}$} & \multicolumn{2}{|l|}{ Error $\varepsilon_{\mu}$} \\
\hline & & Reference & SSC-ENO & SSC & SSC-ENO & SSC \\
\hline 1 & 17 & $1.1028 \cdot 10^{0}$ & $1.1029 \cdot 10^{0}$ & $1.1059 \cdot 10^{0}$ & $1.4009 \cdot 10^{-4}$ & $3.1434 \cdot 10^{-3}$ \\
\hline 2 & 100 & $1.1082 \cdot 10^{0}$ & $1.1088 \cdot 10^{0}$ & $1.1114 \cdot 10^{0}$ & $5.9472 \cdot 10^{-4}$ & $3.2353 \cdot 10^{-3}$ \\
\hline 3 & 200 & $1.1109 \cdot 10^{0}$ & $1.1108 \cdot 10^{0}$ & $1.1069 \cdot 10^{0}$ & $1.7910 \cdot 10^{-4}$ & $4.0323 \cdot 10^{-3}$ \\
\hline 4 & 200 & $1.1256 \cdot 10^{0}$ & $1.1182 \cdot 10^{0}$ & $1.0949 \cdot 10^{0}$ & $7.3967 \cdot 10^{-3}$ & $3.0681 \cdot 10^{-2}$ \\
\hline 5 & 200 & $1.1256 \cdot 10^{0}$ & $1.1097 \cdot 10^{0}$ & $1.1354 \cdot 10^{0}$ & $1.5916 \cdot 10^{-2}$ & $9.8116 \cdot 10^{-3}$ \\
\hline
\end{tabular}


Carlo reference solution with $n_{\mathrm{mc}}=50,000$ samples in Tables 1 and 2 . In most cases, SSC-ENO is more accurate than SSC by an order of magnitude. This improved accuracy is mainly caused by the increased effectiveness of the refinement. To illustrate this mechanism, we introduce a measure for the refinement effectiveness, RE, in terms of the fraction of the refinements that split the discontinuous elements

$$
\mathrm{RE}=\frac{n_{\text {ref, disc }}}{n_{\text {ref }}}
$$

with $n_{\text {ref,disc }}$ the number of times the elements that contain the discontinuities is refined, and $n_{\text {ref }}$ the total number of refinement cycles. The effectiveness given in Table 3 is increased from a minimum of $\mathrm{RE}=42.9 \%$ for SSC to a maximum of $\mathrm{RE}=77.9 \%$ for SSC-ENO. The decreasing difference between SSC-ENO and SSC with increasing $n_{\xi}$ is caused by the simplification in the efficient implementation and the constant value of $n_{\mathrm{s}}$.

\section{Chemical model problem}

The SSC-ENO method is applied to a chemical model problem with a discontinuity developing from two different smooth initial conditions.

\subsection{Initial-value problem}

A simplified version of the Barkley model [5] considered in [20,23] consists of the following ordinary differential equation (ODE)

$$
\frac{\mathrm{d} u}{\mathrm{~d} t}=-u(u-a)(u-b),
$$

with the uncertain initial condition $u(t, \xi)=u_{0}(\xi)$ for $t=0$, and the parameter values $a=-6$ and $b=1$ from [20]. The numerical time integration is performed using the fourth order explicit Runge-Kutta method with a time step of $\Delta t=10^{-3}$. The realizations of (19) approach one of the two stable attractors at $u=-6$ or $u=1$ for negative or positive $u_{0}(\xi)$, respectively. As a consequence, a high gradient forms in parametric space at $u_{0}=0$.

We consider a normal distribution for $u_{0}(\xi)$ with two different mean values, $\mu_{\mathrm{u}_{0}}=0.2$ and $\mu_{\mathrm{u}_{0}}=1$, and a constant coefficient of variation of $\mathrm{CoV}_{\mathrm{u}_{0}}=1$. In Fig. 10, it can be seen that the probability of negative initial values, $P\left(u_{0}<0\right)$, is identical for both cases, such that they have the same asymptotic solution for the mean $\mu_{\mathrm{u}_{\infty}}=\mu_{\mathrm{u}}(t \rightarrow \infty)$

$$
\mu_{\mathrm{u}_{\infty}}=a P\left(u_{0}<0\right)+b P\left(u_{0}>0\right),
$$

and the standard deviation $\sigma_{\mathrm{u}_{\infty}}$

$$
\sigma_{\mathrm{u}_{\infty}}=\sqrt{\left(a-\mu_{\mathrm{u}_{\infty}}\right)^{2} P\left(u_{0}<0\right)+\left(b-\mu_{\mathrm{u}_{\infty}}\right)^{2} P\left(u_{0}>0\right)} .
$$

On the other hand, the transient behavior depends on the mean initial condition $\mu_{\mathrm{u}_{0}}$. We focus, therefore, on resolving the large gradient at $t=1.5$ as well as the smooth transient solution in $t \in T=[0 ; 1.5]$. To that end, the element refinement criterion $e_{j}$ is taken to be the maximum of the refinement measures $e_{t, j}(t)$ that are obtained for each $\Xi_{j}$ at each time step according to

$$
e_{j}=\max _{t \in T} e_{\mathrm{t}, j}(t)
$$

The unbounded parameter range for the normal distribution is treated by truncating the domain beyond the extremes of the

\begin{tabular}{|c|c|c|c|c|c|c|}
\hline \multirow[t]{2}{*}{$n_{\xi}$} & \multirow[t]{2}{*}{$n_{\mathrm{s}}$} & \multicolumn{3}{|c|}{ Standard deviation $\sigma_{\mathrm{u}}$} & \multicolumn{2}{|l|}{$\underline{\text { Error } \varepsilon_{\sigma}}$} \\
\hline & & Reference & SSC-ENO & SSC & SSC-ENO & SSC \\
\hline 1 & 17 & $5.0506 \cdot 10^{-1}$ & $5.0489 \cdot 10^{-1}$ & $5.0305 \cdot 10^{-1}$ & $1.6762 \cdot 10^{-4}$ & $2.0047 \cdot 10^{-3}$ \\
\hline 2 & 100 & $4.9328 \cdot 10^{-1}$ & $4.8444 \cdot 10^{-1}$ & $4.7901 \cdot 10^{-1}$ & $8.8390 \cdot 10^{-3}$ & $1.4268 \cdot 10^{-2}$ \\
\hline 3 & 200 & $4.9275 \cdot 10^{-1}$ & $4.6325 \cdot 10^{-1}$ & $4.5551 \cdot 10^{-1}$ & $2.9503 \cdot 10^{-2}$ & $3.7238 \cdot 10^{-2}$ \\
\hline 4 & 200 & $4.8889 \cdot 10^{-1}$ & $4.1599 \cdot 10^{-1}$ & $4.1673 \cdot 10^{-1}$ & $7.2898 \cdot 10^{-2}$ & $7.2163 \cdot 10^{-2}$ \\
\hline 5 & 200 & $4.8888 \cdot 10^{-1}$ & $3.8789 \cdot 10^{-1}$ & $3.7364 \cdot 10^{-1}$ & $1.0098 \cdot 10^{-1}$ & $1.1524 \cdot 10^{-1}$ \\
\hline
\end{tabular}
Monte Carlo integration points for computing the statistical moments, as proposed in [35].

Table 2

Approximation of the standard deviation by SSC and SSC-ENO with the refinement measure $e_{j}$ and $n_{\xi}=\{1,2,3\}$ compared to the reference solution for the test function. 
Table 3

Refinement effectiveness, RE, by SSC and SSC-ENO with the refinement measure $e_{j}$ and $n_{\xi}=\{1,2,3\}$ for the test function.

\begin{tabular}{llll}
\hline$n_{\xi}$ & $n_{\mathrm{s}}$ & SSC-ENO & SSC \\
\hline 1 & 17 & 0.643 & 0.429 \\
2 & 100 & 0.779 & 0.453 \\
3 & 200 & 0.744 & 0.550 \\
4 & 200 & 0.689 & 0.634 \\
5 & 200 & 0.743 & 0.713 \\
\hline
\end{tabular}

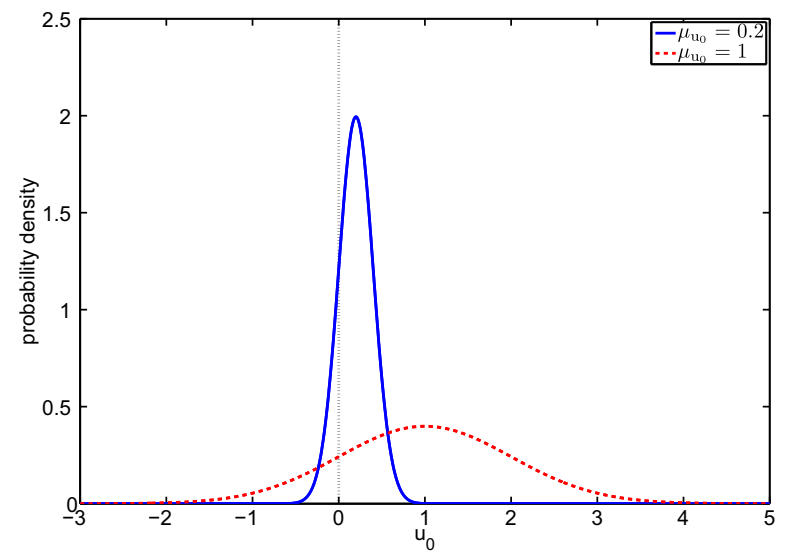

(a) Probability density function

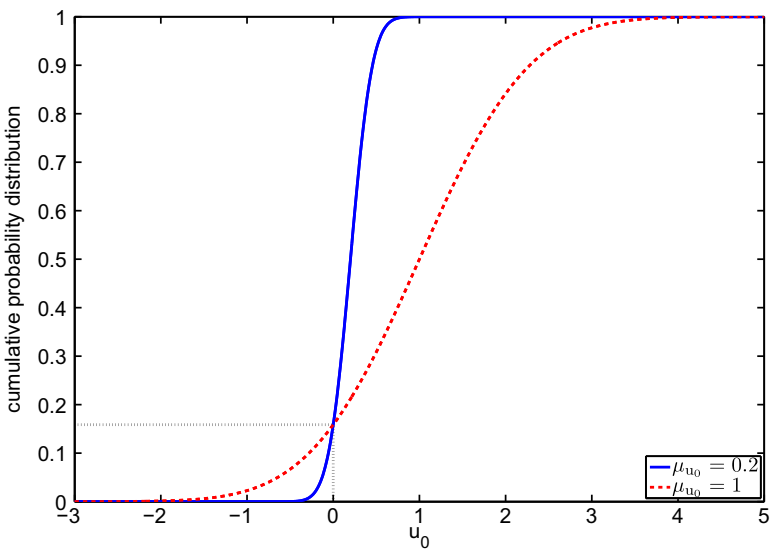

(b) Cumulative probability distribution function

Fig. 10. Probability distribution of the initial condition $u_{0}(\xi)$ for the chemical model problem with $\mu_{\mathrm{u}_{0}}=\{0.2 ; 1\}$.

\subsection{Mean initial value of $\mu_{\mathrm{u}_{0}}=0.2$}

The solution for the mean $\mu_{\mathrm{u}}(t)$ and standard deviation $\sigma_{\mathrm{u}}(t)$ by SSC-ENO is shown for the initial condition $\mu_{\mathrm{u}_{0}}=0.2$ in Fig. 11(a). Initially, $\mu_{\mathrm{u}}(t)$ increases until $t=0.23$ after which it decreases to the asymptotic value of $\mu_{\mathrm{u}_{\infty}}=-0.111$. The formation of the discontinuity leads to a monotonic increase of the standard deviation $\sigma_{\mathrm{u}}(t)$ by more than a factor ten, from the initial $\sigma_{\mathrm{u}_{0}}=0.2$ to $\sigma_{\mathrm{u}_{\infty}}=2.56$ at $t=1.5$. SSC-ENO converges for $n_{\mathrm{s}}=40$ to the converged Monte Carlo reference solution with $n_{\mathrm{mc}}=50,000$ on the whole time interval.

The approximation of the response $u(t, \xi)$ as function of $t$ and $u_{0}(\xi)$ is given in Fig. 11(b) for $n_{\mathrm{s}}=40$. The density of the lines at constant values of $u_{0}$ represents the input probability density of $u_{0}(\xi)$. A relatively small fraction of

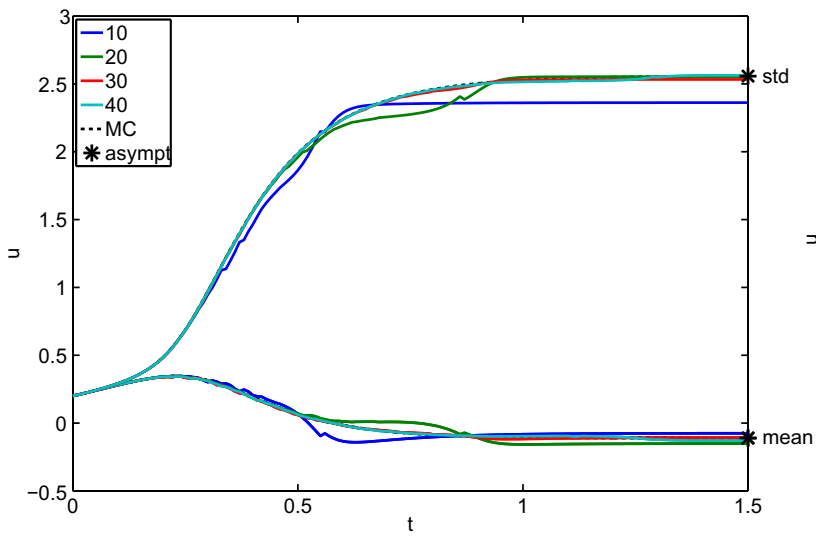

(a) Mean and standard deviation

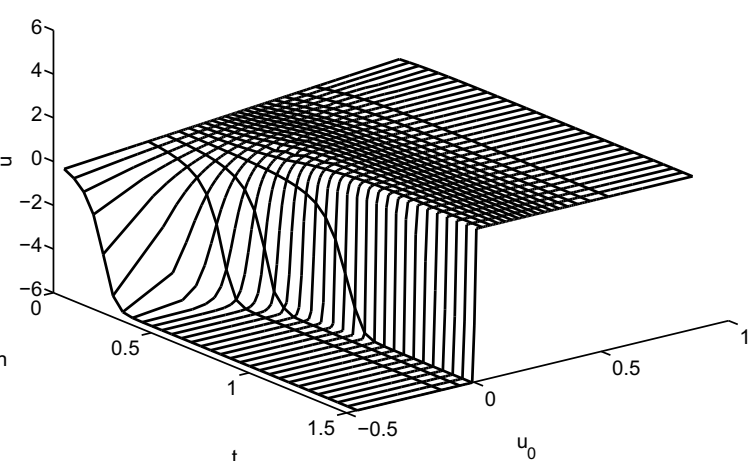

(b) Response surface, $n_{\mathrm{s}}=40$

Fig. 11. SSC-ENO with $n_{\mathrm{s}}=\{10,20,30,40\}$ for the chemical model problem with $\mu_{\mathrm{u}_{0}}=0.2$. 


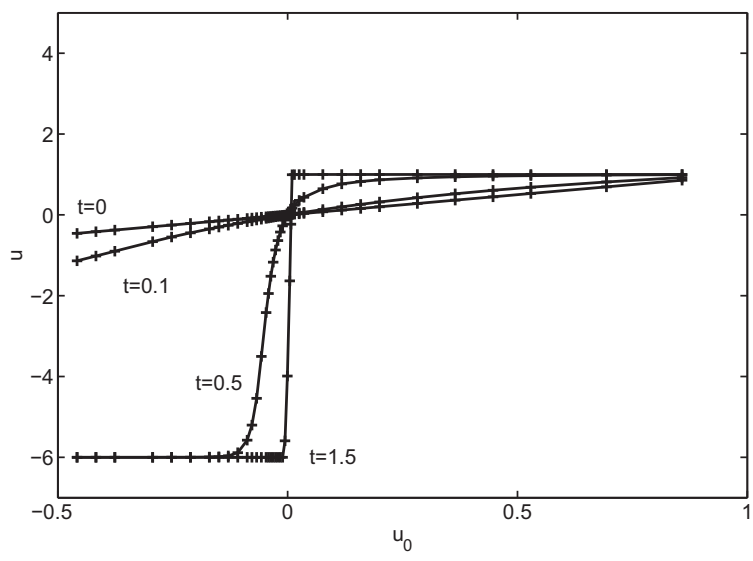

(a) $\mu_{\mathrm{u}_{0}}=0.2$

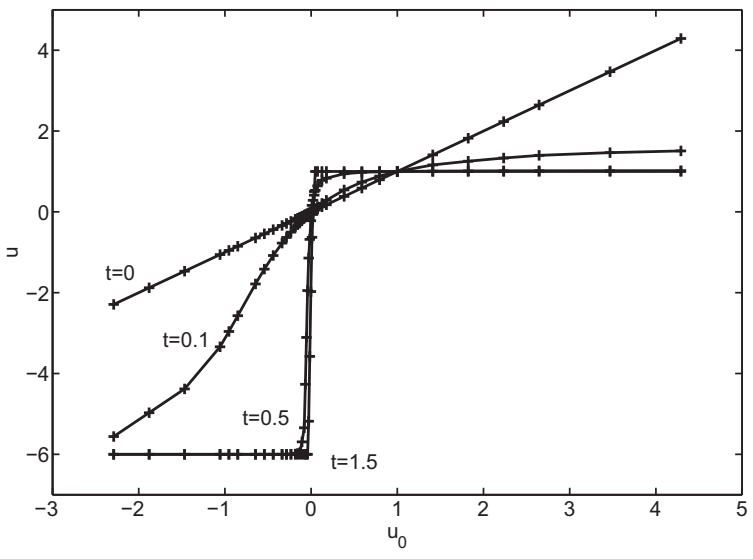

(b) $\mu_{\mathrm{u}_{0}}=1$

Fig. 12. SSC-ENO response surface discretization as function of time at $t=\{0 ; 0.1 ; 0.5 ; 1.5\}$ with $n_{\mathrm{s}}=40$ for the chemical model problem, where the symbols denote the element boundaries.

$P\left(u_{0}<0\right)=15.9 \%$ of the realizations decreases to the stable point $u=-6$. The majority of the response surface increases to $u=1$, which results in the initially increasing transient for $\mu_{\mathrm{u}}(t)$. The eventual decrease of $\mu_{\mathrm{u}}(t)$ is caused by the significantly larger distance of the negative stable point $u=-6$ from the origin compared to the positive attractor $u=1$. The standard deviation gives a monotonically increasing behavior, because the realizations in the relevant part of the parametric space all decrease monotonically at the left of the singularity and increase at the right. The sharp gradient at $t=1.5$ and the smooth transient trajectories are accurately resolved by the combination of first order and higher degree interpolation polynomials in SSC-ENO.

The discretization of the response surface by SSC-ENO with $n_{\mathrm{s}}=40$ is shown in Fig. 12(a) as function of time at $t=\{0 ; 0.1 ; 0.5 ; 1.5\}$. The samples at the element boundaries are denoted by the symbols. The large gradient in parametric space forms at $t=0.5$ for $u_{0}<0$ and approaches its asymptotic location $u_{0}=0$ from below. The refinement criterion (22) successfully resolves this transient drift of the location of the largest gradient using smaller element sizes at the left of $u_{0}=0$, compared to the larger elements in the smoother region for $u_{0}>0$.

\subsection{Mean initial value of $\mu_{\mathrm{u}_{0}}=1$}

The mean, the standard deviation, and the response surface for the second case with $\mu_{\mathrm{u}_{0}}=1$ are given in Fig. 13. In contrast to the case with $\mu_{\mathrm{u}_{0}}=0.2$, the mean $\mu_{\mathrm{u}}(t)$ shows a monotonic decrease to $\mu_{\mathrm{u}_{\infty}}$. The standard deviation $\sigma_{\mathrm{u}}(t)$ also has a different transient with an initial decrease until it starts to increase at $t=0.035$ to $\sigma_{\mathrm{u}_{\infty}}$. This qualitative difference is caused by the decay, at the right of the singularity $u_{0}=0$, of $50 \%$ of the realizations to the positive stable point $u=1$ as shown in Fig. 13(b). SSC-ENO captures this different transient with $n_{\mathrm{s}}=40$ samples. Because of the larger input standard deviation of $\sigma_{\mathrm{u}_{0}}=1$, a relatively sharper gradient develops already at earlier times, which is visible in Fig. 12(b).

\section{Shock tube problem}

A shock tube problem is used here to study the impact of uncertain discontinuity locations on a spatial flow field compared to the deterministic solution.

\subsection{Sod's Riemann problem in a closed shock tube}

The system of hyperbolic Euler equations for one-dimensional unsteady inviscid flow without heat conduction is given in conservation formulation by

$$
\frac{\partial U}{\partial t}+\frac{\partial F(U)}{\partial x}=0
$$

with the state vector $U(x, t)$ and flux vector $F(x, t)$

$$
U=\left(\begin{array}{c}
\rho \\
\rho u \\
\rho E
\end{array}\right), \quad F=\left(\begin{array}{c}
\rho u \\
\rho u^{2}+p \\
\rho u H
\end{array}\right),
$$




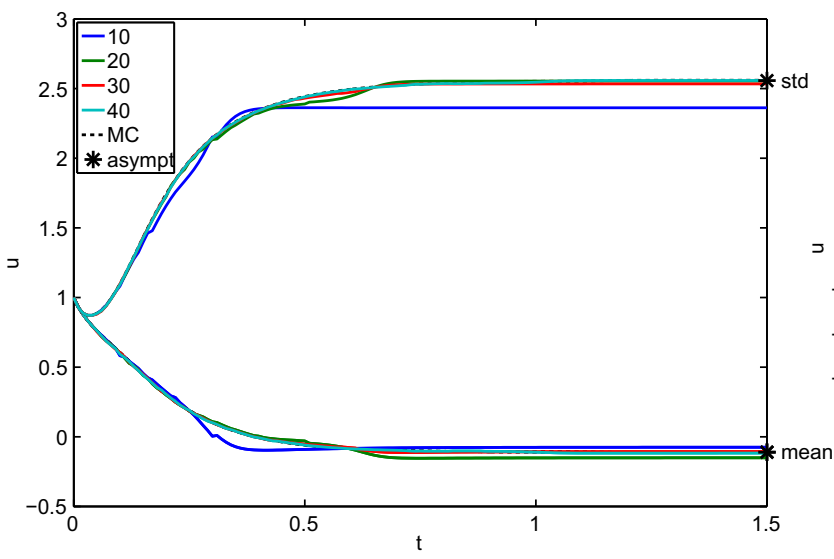

(a) Mean and standard deviation

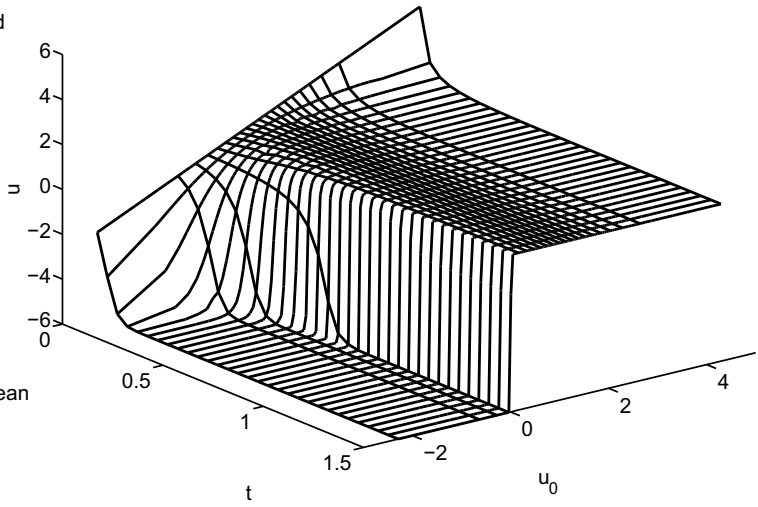

(b) Response surface, $n_{\mathrm{s}}=40$

Fig. 13. SSC-ENO with $n_{\mathrm{s}}=\{10,20,30,40\}$ for the chemical model problem with $\mu_{\mathrm{u}_{0}}=1$.

and initial conditions $U(x, 0)=U_{0}(x)$. For a perfect gas, the density $\rho(x, t)$, velocity $u(x, t)$, static pressure $p(x, t)$, total energy $E(x, t)$, and enthalpy $H(x, t)$ are related as $E=(1 /(\gamma-1)) p / \rho+u^{2} / 2$ and $H=E+p / \rho$, with ratio of specific heats $\gamma=c_{\mathrm{p}} / c_{\mathrm{v}}$ [7]. The initial conditions $U_{0}(x)$ involve Sod's Riemann problem [26] with two uniform states at the left and the right of $x_{0}=0$

$$
\left\{\begin{array} { l } 
{ u _ { \text { left } } = 0 , } \\
{ p _ { \text { left } } = 1 , } \\
{ \rho _ { \text { left } } = 1 , }
\end{array} \quad \left\{\begin{array}{l}
u_{\text {right }}=0, \\
p_{\text {right }}=0.1, \\
\rho_{\text {right }}=0.125
\end{array}\right.\right.
$$

The uncertainty in the pressure $p_{\text {left }} \in[0.9 ; 1.1]$ of the initial left state and the location $x_{0} \in[-0.025 ; 0.025]$ of the initial discontinuity is given by the uniform distribution. The output quantities of interest are the density at $x=0.82$, and $\rho, p$, and $u$ on the entire spatial domain. The problem is here confined to a closed shock tube on a finite spatial domain $x \in[-0.2 ; 2]$ with reflective walls at the boundaries, as considered deterministically in [32]. The Euler equations (23) are solved up to $t=1$ using a second order front tracking method [29,32], which tracks the location of waves in the flow solution and solves local Riemann problems to simulate their interactions. It resolves shock waves and contact surfaces as true discontinuities unaffected by numerical diffusion, which results in sharp jumps in the physical and parametric spaces. Rarefaction waves are discretized by a series of characteristics and second order convergence is obtained using a piecewise linear reconstruction of the rarefaction wave solution. Based on a convergence study, the rarefaction wave is here discretized using $n_{\mathrm{f}}=64$ characteristic fronts.

\subsection{Deterministic solution}

The space-time solution of the deterministic problem is shown in Fig. 14(a) in terms of the wave paths for $n_{\mathrm{f}}=16$. A left running rarefaction wave, a contact discontinuity, and a right running shock wave emanate from the discontinuity in the initial conditions at $x_{0}=0$. The rarefaction wave reflects from the left boundary and interacts with the contact discontinuity in the interior of the domain. The corresponding profiles of the density $\rho$, pressure $p$, and velocity $u$ at $t=1$ are given in Figs. 14 (b)-(d) for $n_{\mathrm{f}}=64$. Next to the shock wave, the contact surface also results in a discontinuity in the density field, while $p$ and $u$ remain continuous in the interaction region.

\subsection{Uncertain density at $x=0.82$}

The effect on the density $\rho$ in the vicinity of the contact discontinuity is resolved for $x=0.82$ and $t=1$ by SSC-ENO using measure $e_{j}$ and $n_{\mathrm{s}}=100$. The response surface approximation for $\rho$ as function of $p_{\text {left }}$ and $x_{0}$ in Fig. 15(a) shows that the test function considered in Section 3 is representative for the solution of hyperbolic conservation laws. The response shows two continuous regions separated by a diagonal discontinuity, which varies in strength and is slightly curved at relatively high values of $p_{\text {left }}$. SSC-ENO leads to a robust approximation and an effective refinement of the discontinuity in Fig. 15(b) with a large ratio between the size of the simplexes that contain the contact surface and the ones that discretize the continuous density variations. This results for the density at $x=0.82$ in a mean of $\mu_{\rho}=0.231$ and a standard deviation of $\sigma_{\rho}=0.0543$, which corresponds to a coefficient of variation of $\operatorname{CoV}_{\rho}=23.5 \%$. 


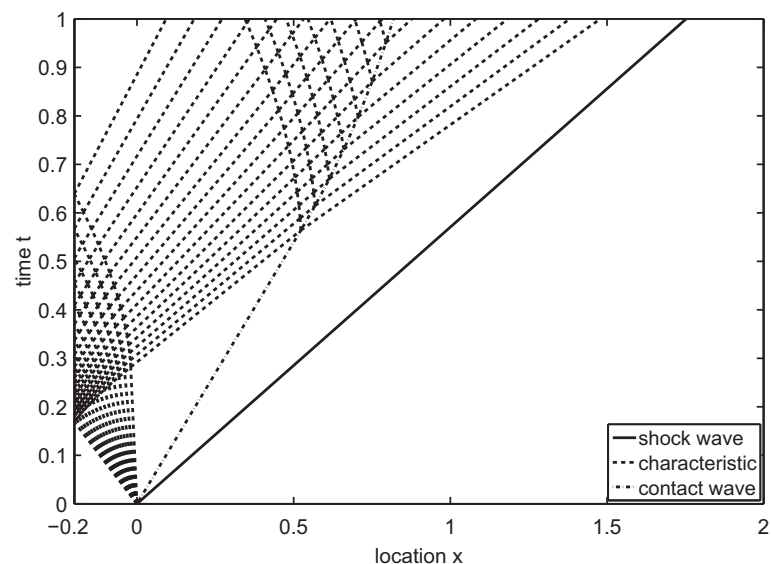

(a) Space-time, $n_{\mathrm{f}}=16$

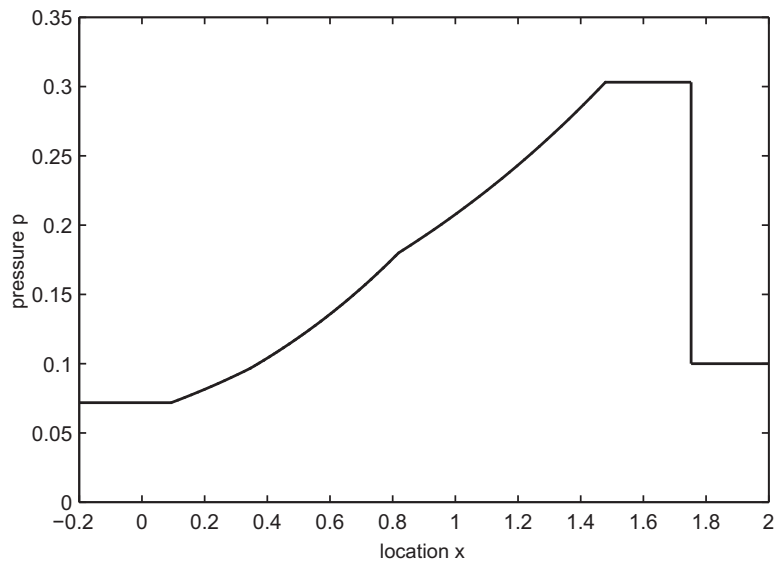

(c) Pressure $p$ at $t=1, n_{\mathrm{f}}=64$

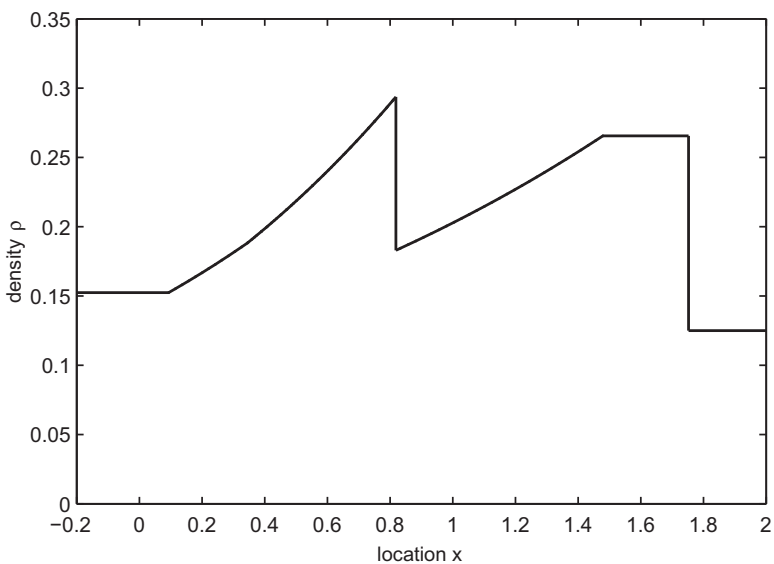

(b) Density $\rho$ at $t=1, n_{\mathrm{f}}=64$

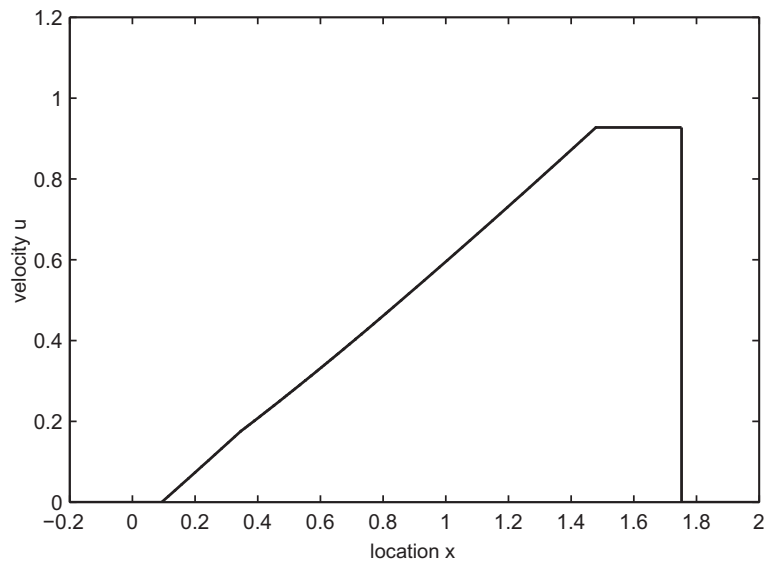

(d) Velocity $u$ at $t=1, n_{\mathrm{f}}=64$

Fig. 14. Sod's Riemann problem in a closed shock tube with deterministic initial conditions.

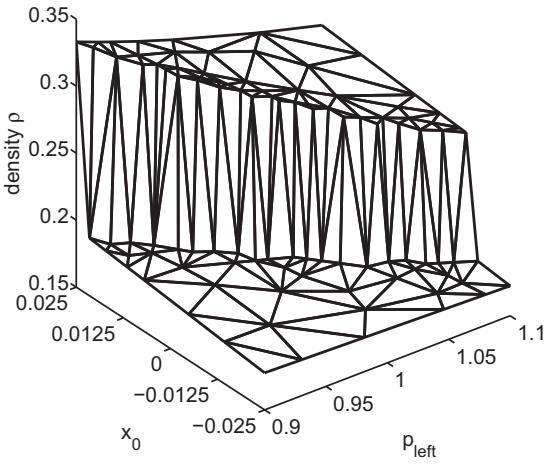

(a) Response surface

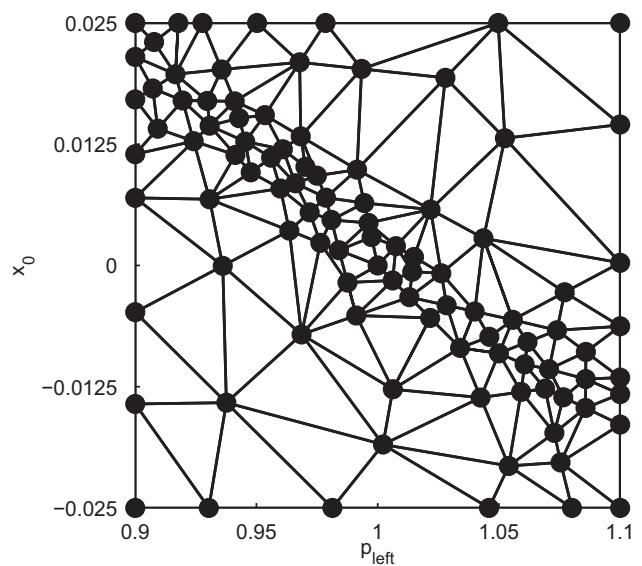

(b) Discretization

Fig. 15. Density $\rho$ at $x=0.82$ and $t=1$ by SSC-ENO with the measure $e_{j}$ and $n_{\mathrm{s}}=100$ for Sod's Riemann problem in a closed shock tube with uncertain $p_{\text {left }}$ and $x_{0}$. 


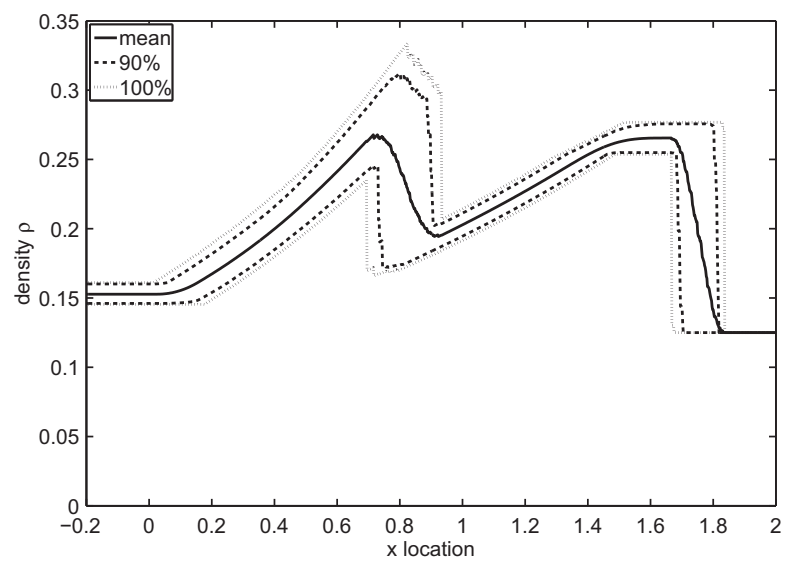

Fig. 16. Mean, and $90 \%$ and $100 \%$ uncertain intervals for the density $\rho$ at $t=1$ by SSC-ENO with the measure $\bar{\Xi}_{j}$ and $n_{\mathrm{s}}=100$ for Sod's Riemann problem in a closed shock tube with uncertain $p_{\text {left }}$ and $x_{0}$.

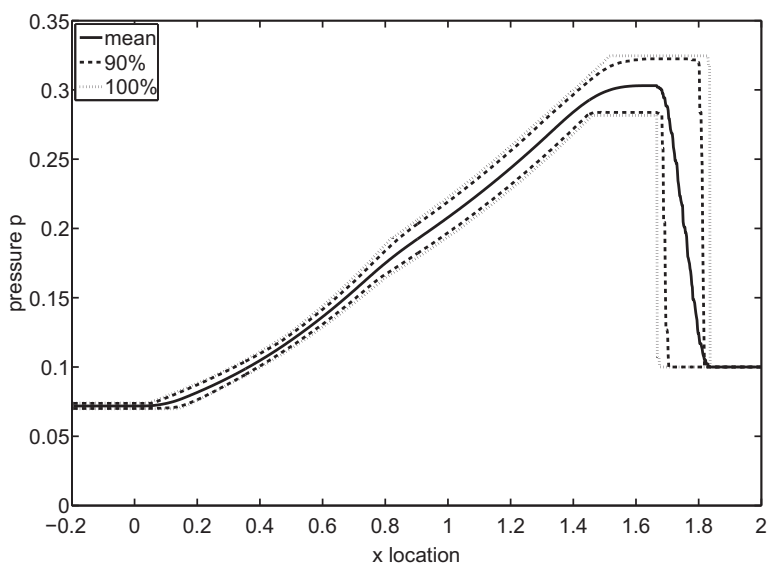

(a) Pressure $p$

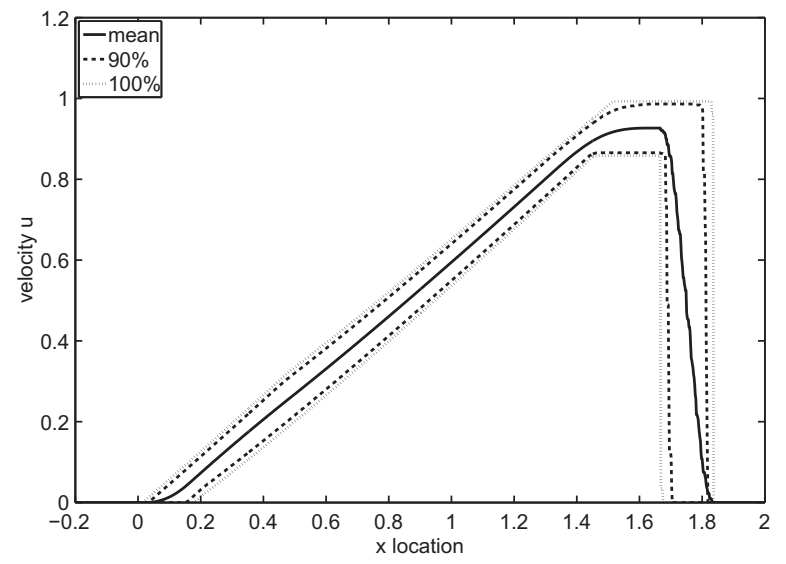

(b) Velocity $u$

Fig. 17. Mean, and $90 \%$ and $100 \%$ uncertain intervals at $t=1$ by SSC-ENO with the measure $\bar{\Xi}_{j}$ and $n_{\mathrm{s}}=100$ for Sod's Riemann problem in a closed shock tube with uncertain $p_{\text {left }}$ and $x_{0}$.

\subsection{Uncertain density, pressure, and velocity fields}

The output uncertainty in the entire density profile on $x \in[-0.2 ; 2]$ is depicted in Fig. 16 in terms of the mean value, and the $90 \%$ and $100 \%$ uncertainty intervals for $n_{\mathrm{s}}=100$. The mean density shows the smearing of the shock and contact waves compared to the deterministic solution, which is caused by the effect of the uncertainty on the location of the discontinuities. The variation in the discontinuity positions is captured by the $100 \%$ interval, which is broadest in these regions and asymmetrical around the mean indicating a highly nonlinear propagation of the uncertainty. The interval around the shock wave shows the robust approximation of SSC-ENO without overshoots at the discontinuity. The varying interval size near the contact surface is caused by the physical variation of the density jump in the interaction region. There is no uncertainty at the right boundary, because the region at the right of the shock wave lies outside the domain of influence of $p_{\text {left }}$ and $x_{0}$.

The corresponding solutions for the pressure $p$ and the velocity $u$ in Fig. 17 are dominated by the uncertainty generated by the shock wave with no major impact of the presence of the contact surface. Refinement measure $\bar{\Xi}_{j}$ is used here, since the discontinuities have different locations in parametric space for each spatial point $x$.

The small steps in the uncertainty interval for the density near the contact discontinuity, in Fig. 16, are caused by the known challenge of representing uncertain discontinuity locations. This is further explored in Fig. 18 for the convergence of the mean and the standard deviation of $\rho$ with $n_{\mathrm{s}}=\{10,20,100\}$. The approximation of the mean for $n_{\mathrm{s}}=10$ contains a smaller number of larger steps in the discontinuous regions. In a non-intrusive approach, the steps are caused by the discontinuity crossing a sampling point and the lack of resolution of the discontinuity location in between the samples. With an increasing number of samples, the solution converges to a smooth representation with a larger number of smaller jumps. 


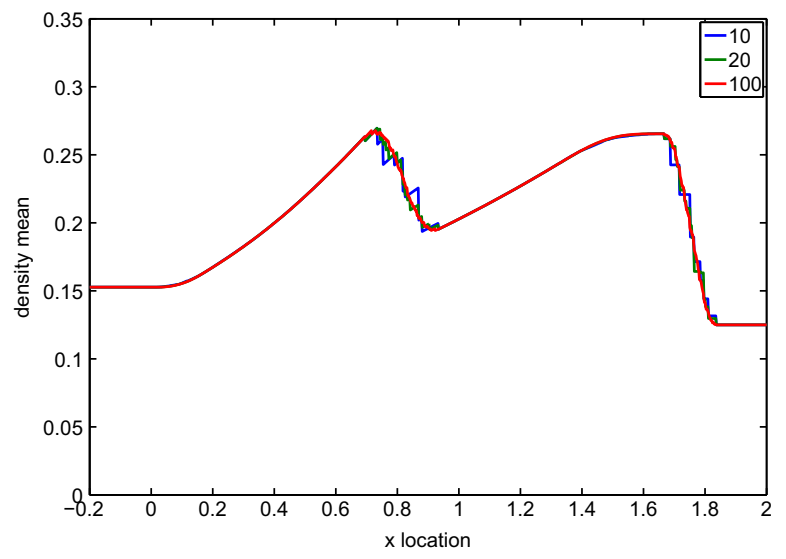

(a) Mean

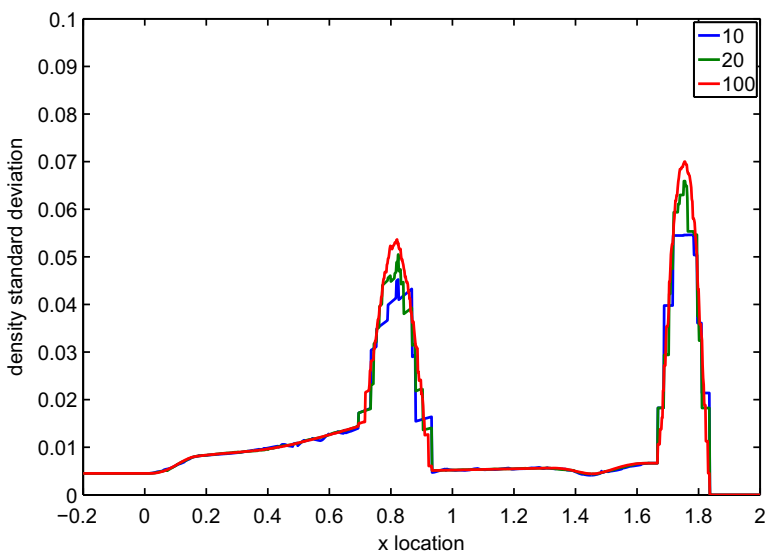

(b) Standard deviation

Fig. 18. Statistical moments of the density $\rho$ at $t=1$ by SSC-ENO with the measure $\bar{\Xi}_{j}$ and $n_{\mathrm{s}}=\{10,20,100\}$ for Sod's Riemann problem in a closed shock tube with uncertain $p_{\text {left }}$ and $x_{0}$.

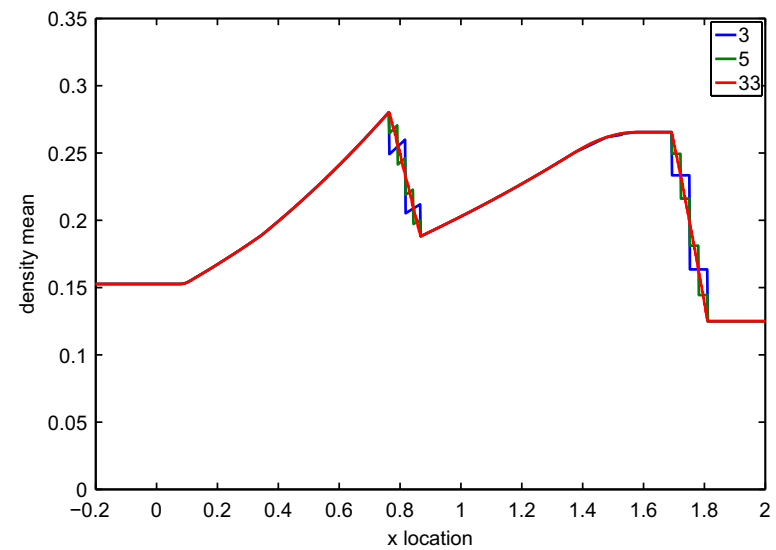

(a) Mean

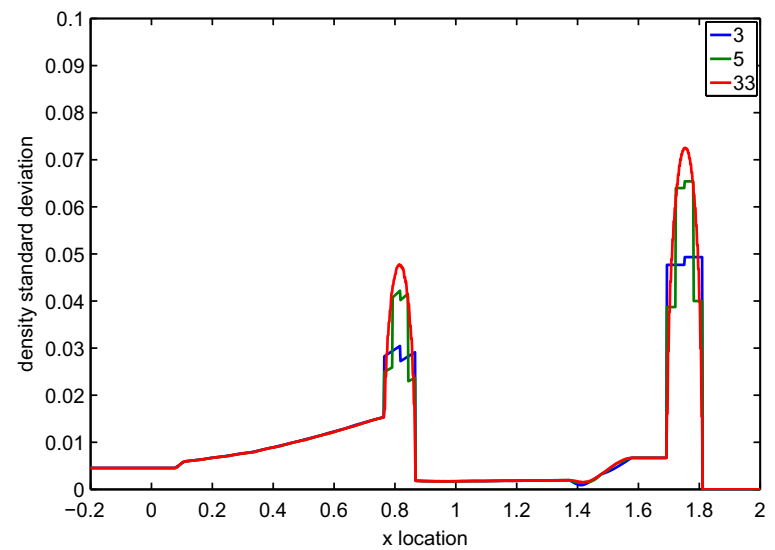

(b) Standard deviation

Fig. 19. Statistical moments of the density $\rho$ at $t=1$ by SSC-ENO with the measure $\bar{\Xi}_{j}$ and $n_{\mathrm{s}}=\{3,5,33\}$ for Sod's Riemann problem in a closed shock tube with uncertain $p_{\text {left }}$.

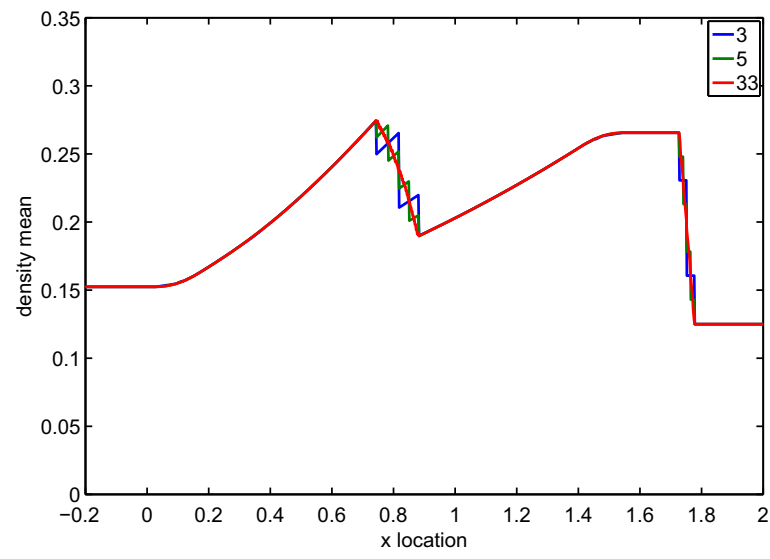

(a) Mean

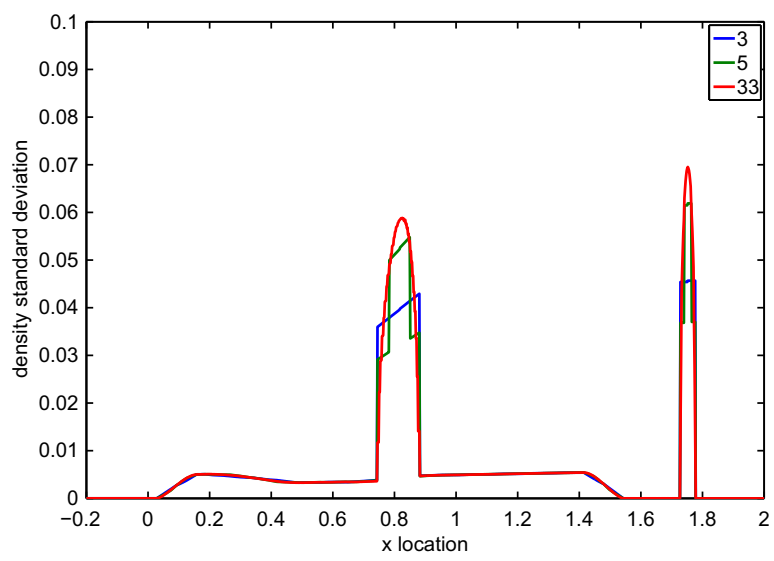

(b) Standard deviation

Fig. 20. Statistical moments of the density $\rho$ at $t=1$ by SSC-ENO with the measure $\bar{\Xi}_{j}$ and $n_{\mathrm{s}}=\{3,5,33\}$ for Sod's Riemann problem in a closed shock tube with uncertain $x_{0}$. 


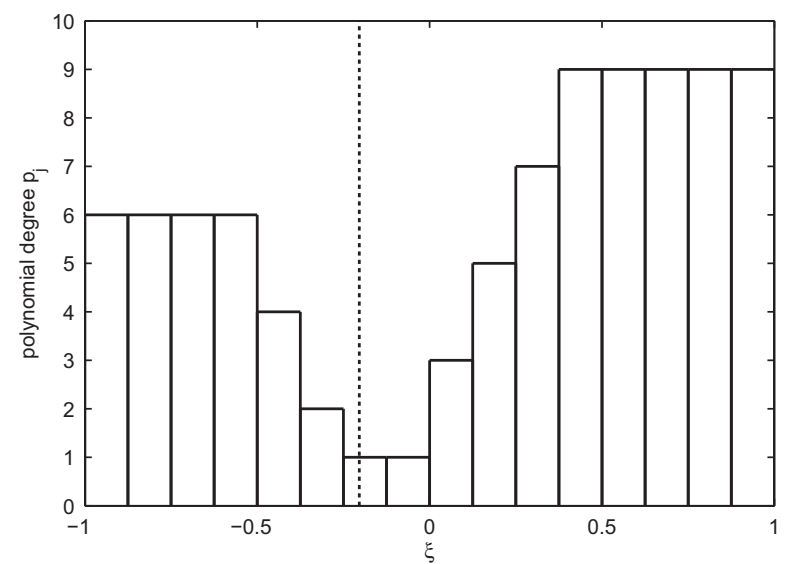

(a) SSC

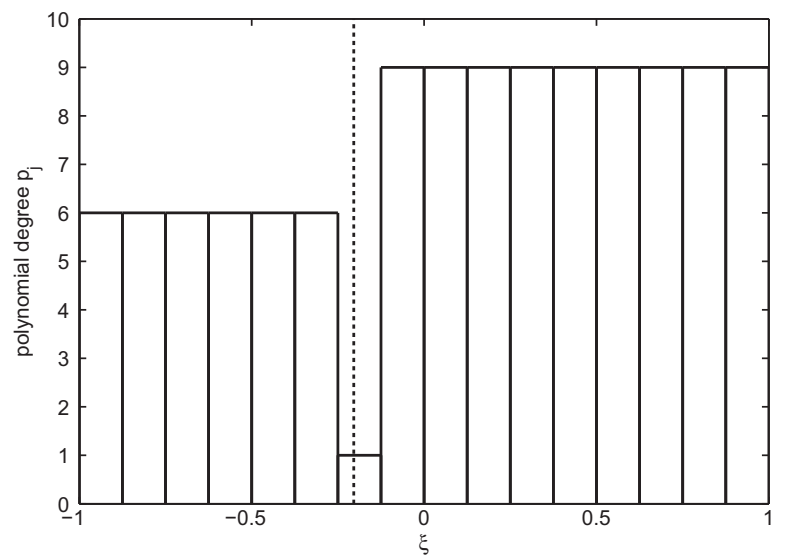

(b) SSC-ENO

Fig. 21. Local polynomial degree $p_{j}$ with the uniform refinement measure $\Xi_{j}$ and $n_{\mathrm{s}}=17$ for the test function, where the vertical dashed line denotes the discontinuity location.

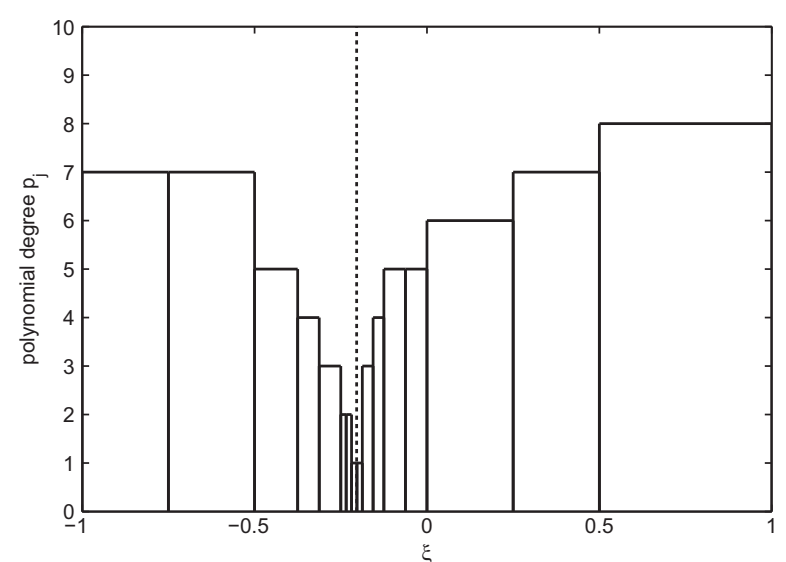

(a) SSC

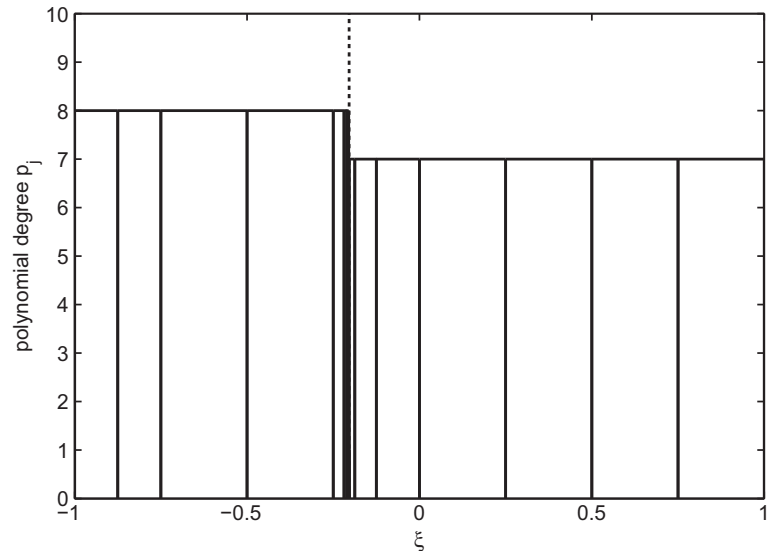

(b) SSC-ENO

Fig. 22. Local polynomial degree $p_{j}$ with the adaptive refinement measure $e_{j}$ and $n_{\mathrm{s}}=17$ for the test function, where the vertical dashed line denotes the discontinuity location.

However, due to the absence of viscosity in the physical problem, the approximation maintains a staircase character which leads to first order accuracy. It also results in the convergence to the maximum standard deviation from below in Fig. 18(b), which causes an underprediction of the maximum standard deviation at underresolved sample sizes.

\subsection{Separate effects of $p_{\text {left }}$ and $x_{0}$}

The individual effects of the uncertainty in $p_{\text {left }}$ and $x_{0}$ on the mean and the standard deviation of the density $\rho$ are given Figs. 19 and 20, respectively. The univariate problems are solved using $n_{s}=\{3,5,33\}$ samples. The number of approximately equidistant steps in the staircase approximation is equal to the number of samples. The uncertain pressure $p_{\text {left }}$ leads to a larger variation in the shock wave location than $x_{0}$, since $p_{\text {left }}$ affects the shock speed. The uncertainty in $x_{0}$ gives a vanishing standard deviation in the uniform flow regions in between the waves, because $x_{0}$ results only in a spatial offset of the initial wave pattern and does not influence the post-states of the Riemann problem.

\section{Conclusions}

Essentially Non-Oscillatory (ENO) type stencil selection is introduced into the Simplex Stochastic Collocation (SSC) method to achieve an accurate approximation of discontinuities in parametric space. The stencil selection for simplex $\Xi_{j}$ chooses the stencil $S_{j}$ with the highest polynomial degree $p_{j}$ that is accepted by the Local Extremum Conserving (LEC) limiter. This 


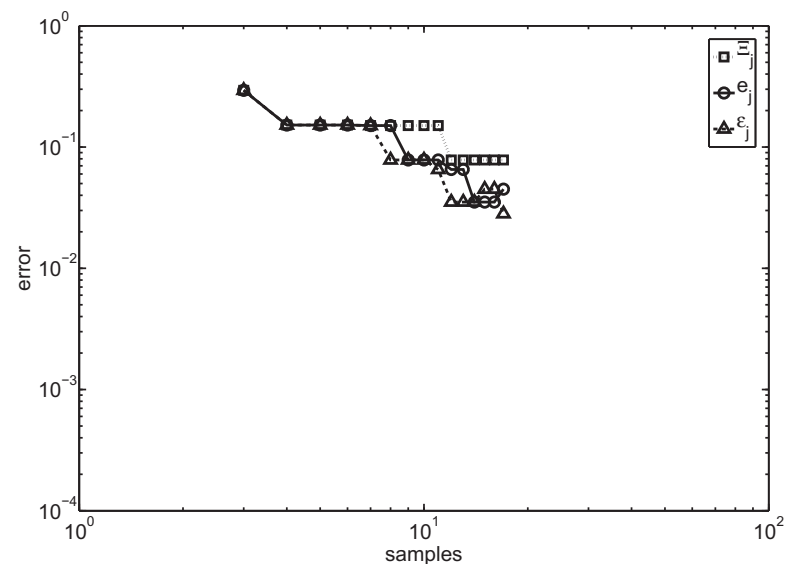

(a) $\mathrm{SSC}$

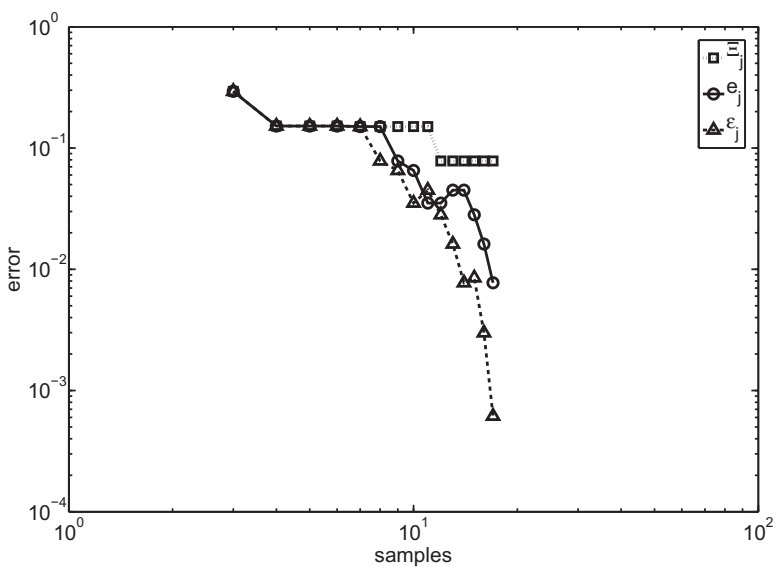

(b) SSC-ENO

Fig. 23. Error convergence for the test function.

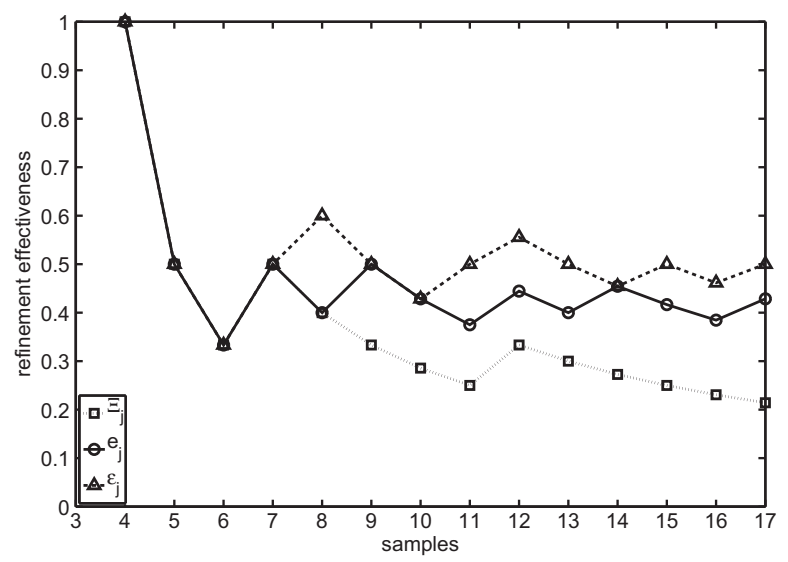

(a) $\mathrm{SSC}$

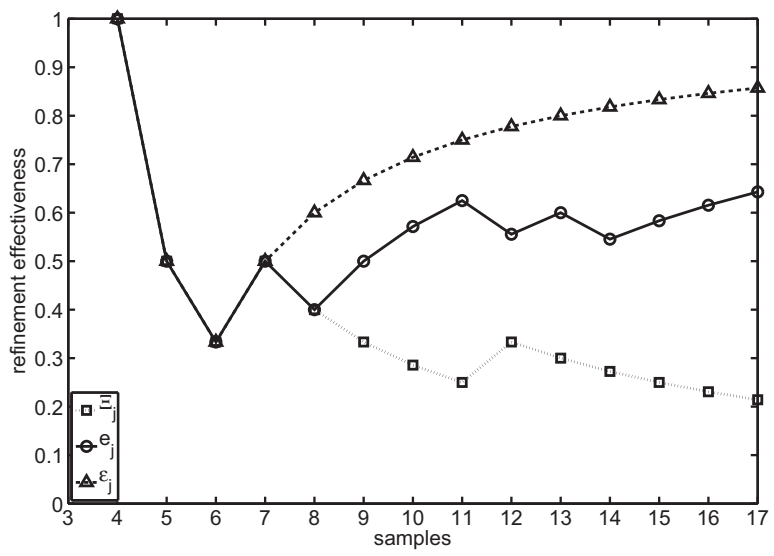

(b) SSC-ENO

Fig. 24. Refinement effectiveness RE (18) for the test function.

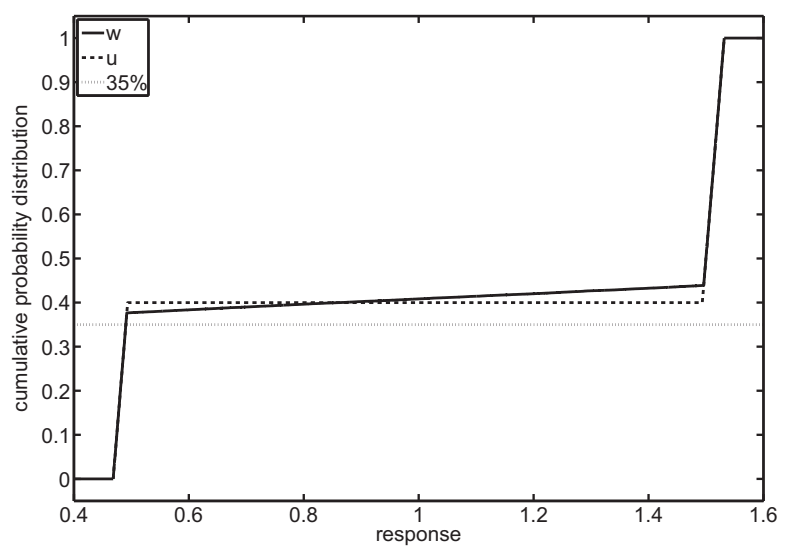

Fig. 25. Cumulative probability distribution function and the 35 th percentile by SSC-ENO with the uniform refinement measure $\Xi_{j}$ and $n_{\mathrm{s}}=17$ for the test function. 


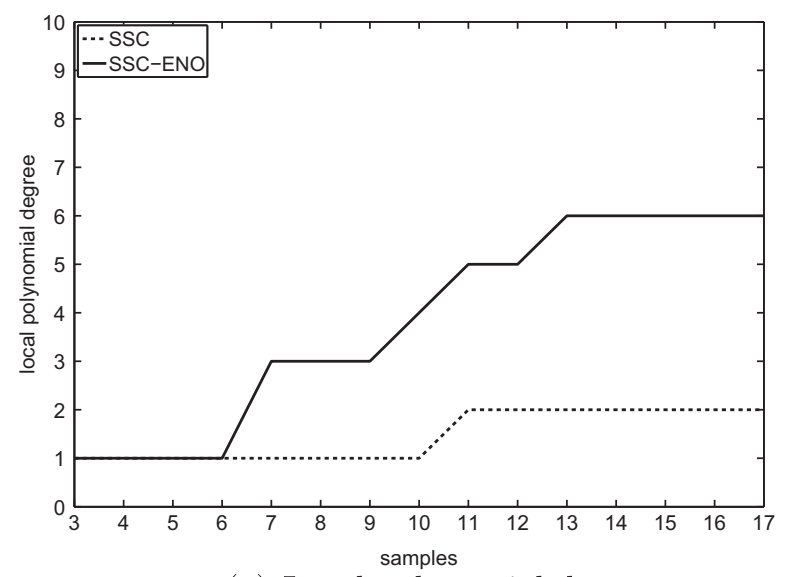

(a) Local polynomial degree

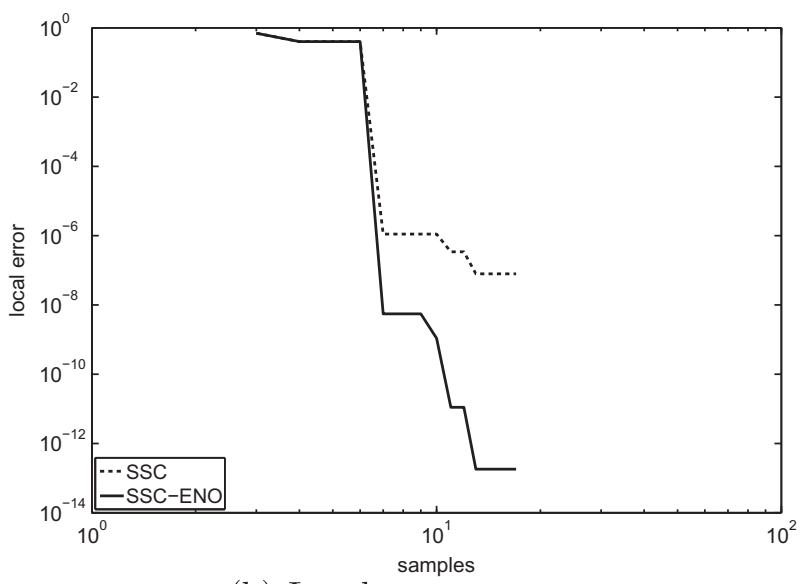

(b) Local error convergence

Fig. 26. Approximation of the 35th percentile with the uniform refinement measure $\bar{\Xi}_{j}$ for the test function.

results in an increase of the local polynomial degree in the smooth regions and a concentration of the refinement in the simplexes that contain the discontinuity. The efficient implementation of the algorithm assigns only nearest neighbor stencils to other simplexes without constructing new stencils or interpolations.

The increased refinement effectiveness of the resulting SSC-ENO method for a discontinuous test function shows that the local polynomial degree is reduced to a linear interpolation in only a thin layer of simplexes that contain the discontinuity. This can lead to a reduction of the size of the discontinuous simplexes by a factor eight and a decrease of the error by two orders of magnitude. It is also observed that the local polynomial degree near a discontinuity can increase from second to sixth order, which can reduce the error in a percentile approximation by six orders of magnitude. A proposed mixed refinement measure successfully distinguishes between discontinuities of different strength.

SSC-ENO is applied to a chemical model problem and a shock tube problem to study the impact of uncertainty on the formation of a discontinuity in time as well as on the location of discontinuities in space. In the chemical model problem, the steepening gradient in time results in an amplification of the input standard deviation by more than a factor ten. The combination of the linear and higher degree polynomials in SSC-ENO accurately resolves both the sharp gradient and the smooth transient trajectories. The adaptive refinement captures the qualitatively different transient behaviors and the transient drift of the location of the largest gradient for $n_{\mathrm{s}}=40$ samples.

The uncertainty in the shock tube problem results in an output coefficient of variation for the density of $23.5 \%$ in the interaction region of the contact and rarefaction wave. The large and asymmetrical uncertainty intervals, near the smeared discontinuities in the mean sense, indicate a robust approximation of the highly nonlinear propagation of the uncertainty in those regions. The staircase approximation of the mean and standard deviation fields in space remains a challenge for representing the solution with more than first order accuracy. In future work, the extension to the Weighted Essentially NonOscillatory (WENO) scheme will also be considered, which has the advantage in spatial discretizations that it improves the order of accuracy by one and leads to a smoother convergence behavior.

\section{Acknowledgments}

This work was supported by the Netherlands Organization for Scientific Research (NWO) and the European Union Marie Curie Cofund Action under Rubicon grant 680-50-1002.

\section{Appendix A. Impact of refinement measures}

The impact of the stencil selection with uniform and adaptive refinement on the error convergence and the percentile approximation through the effect on the local polynomial degree is considered in this appendix for test function (12) with $c_{\text {hat }}=0$ and $n_{\xi}=1$.

\section{A.1. Uniform refinement}

Uniform refinement based on the size $\bar{\Xi}_{j}(10)$ of the elements $\Xi_{j}$ is considered first, in order to separate the effect of the stencil selection on the polynomial degree from its influence on the adaptive refinement. The local polynomial degree $p_{j}$ in the elements $\Xi_{j}$ is given in Fig. 21(a) for SSC with nearest neighbor stencils and $n_{\mathrm{s}}=17$. The location of the discontinuity is denoted by the vertical dashed line. The reduction of the polynomial degree by the LEC limiter to a linear interpolation of the 
samples in the element that contains the step ensures the robust approximation of the jump without overshoots. The polynomial degree gradually increases beyond $p_{j}=1$ on both sides of the element that contains the discontinuity, because larger symmetrical stencils can be built that do not contain the step. Eventually $p_{j}$ reaches its maxima of $p_{j}=6$ and $p_{j}=9$ halfway the two smooth regions, after which it remains constant. In the latter elements, the stencils $S_{j}$ contain all sampling points $\xi_{k}$ on one side of the discontinuity.

In Fig. 21(b), the average polynomial degree of the SSC-ENO interpolation is significantly higher, since all elements that do not contain the discontinuity obtain the maximum polynomial degree. A robust approximation of the jump is maintained, since the degree $p_{j}=1$ in the discontinuous element is not affected. The maximum degree is higher in the larger region at the right of the jump, since it contains more samples than the left half.

\section{A.2. Solution-adaptive refinement}

The polynomial degree in the elements for adaptive refinement measure $e_{j}(8)$ is given in Fig. 22. For SSC, the degree is reduced to $p_{j}=1$ at the jump and increases slowly with distance away from the discontinuity. SSC-ENO leads to a uniform high polynomial degree right up to the discontinuity in both smooth regions. The polynomial degree is reduced from the maximum degree to $p_{j}=1$ in the discontinuous element only. The high polynomial degree in all elements that do not contain the step reduces the refinement measure $e_{j}$ in those elements relatively to $e_{j}$ in the discontinuous element. The sampling is, therefore, more focused in the latter element, which leads to a discontinuous element with a size that is a factor eight smaller for SSC-ENO than for SSC. The uniform polynomial degree in the smooth regions also leads to more uniform sampling in those domains. The maximum degrees are different, and different from the case with uniform refinement, because of the different distribution of the sampling points over the two continuous regions by the adaptive refinement procedure.

The convergence of $\varepsilon_{\mathrm{rms}}(16)$ is shown in Fig. 23 for SSC and SSC-ENO with refinement measures $\bar{\Xi}_{j}, e_{j}$, and $\varepsilon_{j}(8)$. The adaptive refinement measures $e_{j}$ and $\varepsilon_{j}$ result for SSC in only a slightly lower error than uniform refinement with measure $\bar{\Xi}_{j}$. The effectiveness of the adaptive measures is greatly enhanced by SSC-ENO, for which $\varepsilon_{j}$ obtains an order of magnitude lower error than $e_{j}$ and two orders of magnitude improvement compared to $\bar{\Xi}_{j}$ at $n_{\mathrm{s}}=17$. This is a result of the concentration of the refinement in the discontinuous element.

The refinement effectiveness RE (18) as function of the increasing number of samples $n_{\mathrm{s}}$ during the refinement procedure is visualized in Fig. 24. RE of SSC-ENO with measure $\varepsilon_{j}$ approaches 1, since it refines only the discontinuous element in each step up to $n_{\mathrm{s}}=17$, after an initial search for the discontinuity until $n_{\mathrm{s}}=6$. SSC-ENO with $e_{j}$ also reaches a significantly higher effectiveness of $\mathrm{RE}=64.3 \%$ than $\bar{\Xi}_{j}$ with $\mathrm{RE}=21.4 \%$. SSC without stencil selection reduces the equivalent RE for $\varepsilon_{j}$ and $e_{j}$ to $\mathrm{RE}=50.0 \%$ and $\mathrm{RE}=42.9 \%$, respectively. The stencil selection does not affect the refinement for measure $\bar{\Xi}_{j}$.

\section{A.3. Percentile approximation}

In order to quantify the effect of the increased local polynomial degree independently of the improved refinement, an example of the approximation of a percentile is considered for the uniform refinement measure $\bar{\Xi}_{j}$. The solution for the cumulative distribution function (CDF) of $u(\xi)$ is shown in Fig. 25. The discontinuous response surface results for the uniform input distribution of $\xi$ in a non-standard output distribution for $u(\xi)$ with a jump close to the 35th percentile. Since the response for $u(\xi)$ is monotonically increasing and the input distribution is uniform in $[-1,1]$, the approximation at the 35 th percentile corresponds directly with the local response surface approximation at $\xi=-0.3$.

The resulting approximation of the 35th percentile is given in Fig. 26 in terms of the local polynomial degree and the local error. SSC-ENO increases the local degree during the refinement from initially first order to sixth order at $n_{\mathrm{s}}=17$. The polynomial degree does not increase beyond second order for SSC due to the vicinity of the discontinuity. This leads to a reduction of the error in the 35th percentile approximation by SSC-ENO of six orders of magnitude compared to the result of SSC in Fig. 26(b) owing to the higher order approximation of the smooth background function.

\section{References}

[1] R. Abgrall, A simple, flexible and generic deterministic approach to uncertainty quantifications in nonlinear problems: application to fluid flow problems, 5th European Conference on Computational Fluid Dynamics, ECCOMAS CFD, Lisbon, Portugal, 2010.

[2] N. Agarwal, N.R. Aluru, A domain adaptive stochastic collocation approach for analysis of MEMS under uncertainty, J. Comput. Phys. 228 (2009) 76627688.

[3] I. Babuška, R. Tempone, G.E. Zouraris, Galerkin finite elements approximation of stochastic finite elements, SIAM J. Numer. Anal. 42 (2004) 800-825.

[4] I. Babuška, F. Nobile, R. Tempone, A stochastic collocation method for elliptic partial differential equations with random input data, SIAM J. Numer. Anal. 45 (2007) 1005-1034.

[5] D. Barkley, A model for fast computer simulation of waves in excitable media, Physica D 49 (1991) 61-70.

[6] T. Barth, On the propagation of statistical model parameter uncertainty in CFD calculations, Theor. Comput. Fluid Dyn. (2011) DOI: 10.1007/s00162011-0221-2.

[7] A.J. Chorin, J.E. Marsden, A Mathematical Introduction to Fluid Mechanics, Springer, Verlag, New York, 1979.

[8] J. Foo, X. Wan, G.E. Karniadakis, The multi-element probabilistic collocation method (ME-PCM): Error analysis and applications, J. Comput. Phys. 227 (2008) 9572-9595.

[9] M. Gerritsma, J.-B. van der Steen, P. Vos, G. Karniadakis, Time-dependent generalized polynomial chaos, J. Comput. Phys. 229 (2010) $8333-8363$.

[10] R.G. Ghanem, P.D. Spanos, Stochastic Finite Elements: A Spectral Approach, Springer-Verlag, New York, 1991.

[11] D. Gottlieb, D. Xiu, Galerkin method for wave equations with uncertain coefficients, Commun. Comput. Phys. 3 (2008) $505-518$.

[12] A. Harten, High resolution schemes for hyperbolic conservation laws, J. Comput. Phys. 49 (1983) 357-393. 
[13] A. Harten, S. Osher, Uniformly high-order accurate nonoscillatory schemes I, SIAM J. Numer. Anal. 24 (1987) 279-309.

[14] C. Hirsch, Numerical Computation of Internal and External Flows. I: Fundamentals of Computational Fluid Dynamics, second ed., Butterworth, Heinemann, Oxford, 2007.

[15] A. Jameson, Analysis and design of numerical schemes for gas dynamics. I: Artificial diffusion, upwind biasing, limiters and their effect on accuracy and multigrid convergence, Int. J. Comput. Fluid D 4 (1995) 171-218.

[16] X.-D. Liu, S. Osher, T. Chan, Weighted essentially non-oscillatory schemes, J. Comput. Phys. 115 (1994) $200-212$.

[17] X. Ma, N. Zabaras, An adaptive hierarchical sparse grid collocation algorithm for the solution of stochastic differential equations, J. Comput. Phys. 228 (2009) 3084-3113.

[18] O.P. Le Maître, O.M. Knio, H.N. Najm, R.G. Ghanem, Uncertainty propagation using Wiener-Haar expansions, J. Comput. Phys. 197 (2004) 28-57.

[19] O.P. Le Maître, H.N. Najm, R.G. Ghanem, O.M. Knio, Multi-resolution analysis of Wiener-type uncertainty propagation schemes, J. Comput. Phys. 197 (2004) 502-531.

[20] O.P. Le Maître, H.N. Najm, P.P. Pébay, R.G. Ghanem, O.M. Knio, Multi-resolution-analysis scheme for uncertainty quantification in chemical systems, SIAM J. Sci. Comput. 29 (2007) 864-889.

[21] O.P. Le Maître, L. Mathelin, O.M. Knio, M.Y. Hussaini, Asynchronous time integration for polynomial chaos expansion of uncertain periodic dynamics, Discret. Contin. Dyn. S. 28 (2010) 199-226.

[22] G. Poëtte, B. Després, D. Lucor, Uncertainty quantification for systems of conservation laws, J. Comput. Phys. 228 (2009) $2443-2467$.

[23] M.T. Reagan, H.N. Najm, B.J. Debusschere, O.P. Le Maître, O.M. Knio, R.G. Ghanem, Spectral stochastic uncertainty quantification in chemical systems, Combust. Theoret. Model. 8 (2004) 607-632.

[24] C.-W. Shu, S. Osher, Efficient implementation of essentially non-oscillatory shock-capturing schemes, J. Comput. Phys. 77 (1988) $439-471$.

[25] C.-W. Shu, S. Osher, Efficient implementation of essentially non-oscillatory shock-capturing schemes II, J. Comput. Phys. 83 (1989) 32-78.

[26] G.A. Sod, A survey of several finite difference methods for systems of nonlinear hyperbolic conservation laws, J. Comput. Phys. 27 (1978) 1-31.

[27] J. Tryoen, O. Le Maître, M. Ndjinga, A. Ern, Intrusive Galerkin methods with upwinding for uncertain nonlinear hyperbolic systems, J. Comput. Phys. 229 (2010) 6485-6511.

[28] X. Wan, G.E. Karniadakis, An adaptive multi-element generalized polynomial chaos method for stochastic differential equations, J. Comput. Phys. 209 (2005) 617-642.

[29] J.A.S. Witteveen, B. Koren, P.G. Bakker, An improved front tracking method for the Euler equations, J. Comput. Phys. 224 (2007) $712-728$.

[30] J.A.S. Witteveen, H. Bijl, Effect of randomness on multi-frequency aeroelastic responses resolved by unsteady adaptive stochastic finite elements, J. Comput. Phys. 228 (2009) 7025-7045.

[31] J.A.S. Witteveen, H. Bijl, A TVD uncertainty quantification method with bounded error applied to transonic airfoil flutter, Commun. Comput. Phys. 6 (2009) 406-432.

[32] J.A.S. Witteveen, Second order front tracking for the Euler equations, J. Comput. Phys. 229 (2010) 2719-2739.

[33] J.A.S. Witteveen, H. Bijl, Transonic velocity fluctuations simulated using extremum diminishing uncertainty quantification based on inverse distance weighting, Theoret. Comput. Fluid Dyn. 26 (2012) 459-479.

[34] J.A.S. Witteveen, G. Iaccarino, Simplex stochastic collocation with random sampling and extrapolation for nonhypercube parametric spaces, SIAM J. Sci. Comput. 34 (2012) A814-A838.

[35] J.A.S. Witteveen, G. Iaccarino, Refinement criteria for simplex stochastic collocation with local extremum diminishing robustness, SIAM J. Sci. Comput. 34 (2012) A1522-A1543.

[36] D. Xiu, G.E. Karniadakis, The Wiener-Askey polynomial chaos for stochastic differential equations, SIAM J. Sci. Comput. 24 (2002) 619-644.

[37] D. Xiu, J.S. Hesthaven, High-order collocation methods for differential equations with random inputs, SIAM J. Sci. Comput. 27 (2005) $1118-1139$. 